\title{
A UNIQUE PHENOMENON OF THE PANDEMIC 2020: Mortality among COVID-19 Patients is the Lowest on Sundays and Mondays.
}

Dr. Mikhail Teppone.

\author{
Dr. Mikhail Teppone, \\ Orcid N: 0000-0002-5366-3188 \\ Medical Director, Nano City Holdings Berhad, \\ No. 1, Jalan Sungai Jeluh 32/192, \\ Shah Alam, 40460, Selangor, Malaysia. \\ Email: mikhail.teppone@gmail.com
}

Dec. 15,2020

\section{Abstract.}

Background. The Weekly Mortality Cycle among CoViD-19 patients has been studied.

Methods. Mortality data obtained from the 'Worldometer' website were analyzed with a comparison of absolute values, percentages and p-value.

Results. For patients suffering from CoViD-19, the most favorable or the safest days of the week were Sundays and Mondays.

Conclusion. The weekly cycle with decreased mortality on Sundays and Mondays is a unique phenomenon observed among victims of CoViD-19. Presumably the decreased mortality on certain days of the week was related to the optimized therapeutic protocols used on the "safest days". If the factors, which reduced mortality on certain days of the week could be identified, their positive effect should be spread to other days of the week to decrease mortality among patients suffering from CoViD-19.

Keywords. CoViD-19, Mortality, Weekly Cycle, Weekly Mortality Cycle, Pandemic.

Abbreviation. HLM - is a ratio between a number of deaths on the day with the highest mortality and a number of deaths on the day with the lowest mortality.

Abbreviations for the days of the week were used in all tables and in the certain places of the main text: 'Sun' means Sunday(s), 'Mon' - Monday/s, 'Tue' - Tuesday/s, 'Wed' - Wednesday/s, 'Thu' Thursday/s, 'Fri' - Friday/s and 'Sat' - Saturday/s.

\footnotetext{
* Professor Igor A. Gundarov, a Doctor of Medicine, a specialist in the field of epidemiology and medical statistics, comments this study at the Section \# 11.
} 


\section{Introduction.}

On December 31, 2019, Wuhan Municipal Health Commission announced a pneumonia epidemic. ${ }^{1}$ On the same day the WHO's China Country Office was informed of the cases of pneumonia of unknown cause detected in Wuhan City, Hubei Province of China. ${ }^{2}$

On January 3, 2020, the first complete genome of the novel coronavirus (2019-nCoVs) was identified. Several detection tests have been developed by Chinese Center for Disease Control and Prevention. ${ }^{3}$ On February 11, 2020, a new disease was named "the coronavirus disease 2019" or CoViD-19. ${ }^{4}$ On March 11, 2020, World Health Organization declared CoViD-19 pandemic. $^{5}$

\section{Mortality Among People Infected with SARS-CoV-2 and its Dynamic.}

Mortality rate is one of the most important parameters of any infectious disease. So, collection of the date dealing with the current pandemic should be done with great care otherwise coronavirus mortality overestimation can lead to wrong decisions. ${ }^{6}$

In the first report provided by a group of experts from Imperial College London, there were 41 confirmed cases with 2 deaths; the estimated number of infected people was $1,723,{ }^{7}$ (the estimated mortality rate was around $-0.12 \%$ ). In the second report provided by the same group of experts, there were 440 confirmed cases with 9 deaths, and the estimated number of infected people was around $4000,{ }^{8}$ (the estimated mortality rate was around $-0.23 \%$ ). The most pessimistic prognosis was predicted by the same group of scientists on March 18, 2020. ${ }^{9}$

According to the subsequent studies, the mortality rate among SARS-CoV-2 infected people was less than $1 \%,^{10}$ and could vary around $0.3-0.5 \% .^{11}$

Objective: To study the dynamic of weekly mortality rate among people infected with SARS-CoV-2.

Methods: The database of the "Worldwide Daily Mortality" due to CoViD-19 was collected from the 'Worldometer' website ${ }^{12}$ on December 7, 2020, and then presented in Table. 1. The database of the "Worldwide Daily New Cases" infected with SARS-CoV-2 were collected from the same source, but that table was omitted in the manuscript. Forty weeks were analysed (01.03.30-05.12.20).

For each week, weekly mortality and weekly new cases were calculated using simple addition of data for seven days, from Sunday to Saturday. Weekly mortality rate was estimated with dividing "weekly mortality" by "weekly new cases" for each week (Tab. 2). The dynamics of weekly mortality is shown in Figure 1. 
Results: According to the current study, the highest mortality was observed from the mid of March to the mid of May, with the highest rate (9.36\%) during the week No. 7 (April 12-18), when the clinical trials in the group of people infected with SARS-CoV-2 were in the initial phase yet.

Table 1.

Global Mortality Due to CoViD-19 from January 24 to December 5, 2020 [collected on December 7, 2020].

\begin{tabular}{|c|c|c|c|c|c|c|c|c|c|}
\hline week, \# & Sun & Mon & Tue & Wed & Thu & Fri & Sat & Comments & HLR \\
\hline & & & & & & $* * 16$ & 15 & $* *$ Jan 24 & \\
\hline $\mathrm{i}$ & 24 & 26 & 26 & 38 & 43 & 46 & $* 45$ & $*$ Feb 1 & 1.917 \\
\hline ii & 58 & 64 & 66 & 73 & 73 & 86 & 89 & & 1.534 \\
\hline iii & 97 & 108 & 97 & 146 & 122 & 143 & 143 & & 1.505 \\
\hline iv & 106 & 98 & 136 & 117 & 121 & 113 & 100 & & 1.388 \\
\hline $\mathrm{v}$ & 158 & 81 & 64 & 37 & 58 & 65 & 54 & & 4.270 \\
\hline No. 1 & $* 73$ & 67 & 85 & 83 & 102 & 106 & 106 & * Mar 1 & 1.582 \\
\hline 2 & 229 & 197 & 277 & 333 & 358 & 452 & 421 & & 2.294 \\
\hline 3 & 708 & 655 & 837 & 1001 & 1126 & 1417 & 1666 & & 2.544 \\
\hline 4 & 1690 & 1998 & 2586 & 2642 & 3118 & 3551 & 3847 & & 2.276 \\
\hline 5 & 3498 & 4289 & 4754 & $* 5349$ & 6400 & 6197 & 6307 & * Apr 1 & 1.830 \\
\hline 6 & 5260 & 5761 & 7839 & 6928 & 7822 & 7246 & 6405 & & 1.490 \\
\hline 7 & 5772 & 5786 & 7552 & 8263 & 7016 & 8534 & 6701 & & 1.479 \\
\hline 8 & 5045 & 5740 & 7362 & 6697 & 6862 & 6487 & 6223 & & 1.459 \\
\hline 9 & 3945 & 4701 & 7000 & 6675 & 5909 & *5884 & 5665 & * May 1 & 1.774 \\
\hline 10 & 3807 & 4307 & 5950 & 6773 & 5773 & 5634 & 4428 & & 1.779 \\
\hline 11 & 4447 & 3700 & 5765 & 5269 & 5402 & 5234 & 4477 & & 1.558 \\
\hline 12 & 3717 & 3616 & 4712 & 4764 & 5139 & 4887 & 4333 & & 1.421 \\
\hline 13 & 3312 & 3516 & 3900 & 5365 & 4783 & 4968 & 4212 & & 1.620 \\
\hline 14 & 3444 & $* 3239$ & 4779 & 5024 & 5749 & 5040 & 4455 & $*$ Jun 1 & 1.775 \\
\hline 15 & 3670 & 3350 & 4866 & 5280 & 5139 & 4789 & 4431 & & 1.576 \\
\hline 16 & 3502 & 3599 & 6665 & 5386 & 5367 & 5252 & 4599 & & 1.903 \\
\hline 17 & 3595 & 4039 & 5486 & 5208 & 5398 & 4977 & 4726 & & 1.526 \\
\hline 18 & 3684 & 3694 & 5142 & $* 4969$ & 5299 & 5310 & 4707 & * Jul 1 & 1.441 \\
\hline 19 & 3782 & 3692 & 5602 & 5696 & 5654 & 5602 & 5150 & & 1.543 \\
\hline 20 & 4380 & 3920 & 5682 & 5980 & 5967 & 5762 & 5252 & & 1.526 \\
\hline 21 & 4478 & 4123 & 5827 & 7314 & 6479 & 6356 & 5848 & & 1.774 \\
\hline 22 & 4445 & 4253 & 5680 & 7073 & 6839 & 6493 & $* 5717$ & * Aug 1 & 1.663 \\
\hline 23 & 4577 & 4470 & 6341 & 7049 & 6644 & 6602 & 5771 & & 1.577 \\
\hline 24 & 4929 & 4540 & 6513 & 6921 & 6720 & 6128 & 5675 & & 1.524 \\
\hline 25 & 4706 & 4405 & 6335 & 6779 & 6357 & 6213 & 5547 & & 1.539 \\
\hline 26 & 4598 & 4325 & 6202 & 6466 & 6223 & 5837 & 5497 & & 1.495 \\
\hline 27 & 4353 & 4346 & $* 5978$ & 6457 & 6004 & 5863 & 5003 & * Sep 1 & 1.486 \\
\hline 28 & 4247 & 3872 & 4429 & 6230 & 6021 & 5792 & 5116 & & 1.609 \\
\hline 29 & 4002 & 4264 & 6000 & 6007 & 5412 & 5453 & 5200 & & 1.501 \\
\hline 30 & 3992 & 3810 & 5364 & 6048 & 5558 & 5462 & 5101 & & 1.587 \\
\hline 31 & 3795 & 3536 & 5498 & 5836 & $* 5623$ & 5622 & 4874 & * Oct 1 & 1.650 \\
\hline 32 & 4123 & 4270 & 5576 & 5938 & 6431 & 5894 & 5267 & & 1.560 \\
\hline 33 & 3974 & 3782 & 5002 & 6101 & 6119 & 6226 & 5720 & & 1.646 \\
\hline 34 & 4110 & 4440 & 6190 & 6856 & 6502 & 6555 & 5747 & & 1.668 \\
\hline 35 & 4659 & 5113 & 7055 & 7167 & 7170 & 7521 & 6665 & & 1.614 \\
\hline 36 & $* 5480$ & 5761 & 8250 & 9182 & 8869 & 9260 & 7722 & * Nov 1 & 1.690 \\
\hline 37 & 6129 & 6748 & 9358 & 10193 & 9687 & 10003 & 9226 & & 1.663 \\
\hline 38 & 7117 & 7629 & 10578 & 11283 & 11068 & 11169 & 9332 & & 1.585 \\
\hline 39 & 7829 & 8170 & 11786 & 12286 & 11281 & 10832 & 9523 & & 1.569 \\
\hline No. 40 & 7585 & 8462 & $* 11943$ & 12516 & 12834 & 12166 & 10191 & $* \operatorname{Dec} 1$ & 1.692 \\
\hline
\end{tabular}

* A first day of each month.

** January 24, 2020 was the first day mentioned in the database of the 'Worldometer' website. 
Table 2.

Weekly deaths, weekly new cases and mortality rate; Forty weeks were analysed (01.03.20-05.12.20).

\begin{tabular}{|c|c|c|c|c|c|c|c|c|c|}
\hline \multicolumn{2}{|c|}{$\begin{array}{l}\text { Week Number } \\
\text { and dates }\end{array}$} & $\begin{array}{l}\text { Weekly } \\
\text { Deaths }\end{array}$ & $\begin{array}{c}\text { Weekly New } \\
\text { Cases }\end{array}$ & $* \mathrm{MR}$ & \multicolumn{2}{|c|}{$\begin{array}{c}\text { Week Number } \\
\text { and dates }\end{array}$} & $\begin{array}{l}\text { Weekly } \\
\text { Deaths }\end{array}$ & $\begin{array}{l}\text { Weekly New } \\
\text { Cases }\end{array}$ & *MR \\
\hline 1 & 01.03-07.03 & 622 & 27030 & 2.30 & 21 & $19.07-25.07$ & 40425 & 1788346 & 2.26 \\
\hline 2 & $08.03-14.03$ & 2267 & 80847 & 2.80 & 22 & $26.07-01.08$ & 40500 & 1831624 & 2.21 \\
\hline 3 & $15.03-21.03$ & 7410 & 192895 & 3.84 & 23 & $02.08-08.08$ & 41454 & 1800749 & 2.30 \\
\hline 4 & $22.03-28.03$ & 19432 & 359549 & 5.40 & 24 & $09.08-15.08$ & 41426 & 1853586 & 2.23 \\
\hline 5 & $29.03-04.04$ & 36794 & 502534 & 7.32 & 25 & $16.08-22.08$ & 40342 & 1771117 & 2.28 \\
\hline 6 & 05.04-11.04 & 47261 & 550941 & 8.58 & 26 & $23.08-29.08$ & 39148 & 1808783 & 2.16 \\
\hline 7 & $12.04-18.04$ & 49624 & 530089 & 9.36 & 27 & $30.08-05.09$ & 38004 & 1911797 & 1.99 \\
\hline 8 & $19.04-25.04$ & 44416 & 558829 & 7.95 & 28 & 06.09-12.09 & 35707 & 1904865 & 1.87 \\
\hline 9 & $26.04-02.05$ & 39779 & 545512 & 7.29 & 29 & 13.09-19.09 & 36338 & 2043230 & 1.78 \\
\hline 10 & 03.05-09.05 & 36672 & 606805 & 6.04 & 30 & $20.09-26.09$ & 35335 & 2042437 & 1.73 \\
\hline 11 & $10.05-16.05$ & 34294 & 615151 & 5.57 & 31 & $27.09-03.10$ & 34784 & 2072676 & 1.68 \\
\hline 12 & $17.05-23.05$ & 31168 & 688057 & 4.53 & 32 & $04.10-10.10$ & 37499 & 2290257 & 1.64 \\
\hline 13 & $24.05-30.05$ & 30056 & 769786 & 3.90 & 33 & $11.10-17.10$ & 36924 & 2491069 & 1.48 \\
\hline 14 & $31.05-06.06$ & 31730 & 844284 & 3.76 & 34 & $18.10-24.10$ & 40400 & 2979525 & 1.36 \\
\hline 15 & $07.06-13.06$ & 31525 & 902187 & 3.49 & 35 & $25.10-31.10$ & 45350 & 3438119 & 1.32 \\
\hline 16 & $14.06-20.06$ & 34370 & 1026292 & 3.35 & 36 & $01.11-07.11$ & 54524 & 3874278 & 1.41 \\
\hline 17 & $21.06-27.06$ & 33429 & 1170067 & 2.86 & 37 & 18.11-14.11 & 61344 & 4074584 & 1.51 \\
\hline 18 & $28.06-04.07$ & 32805 & 1330213 & 2.47 & 38 & $15.11-21.11$ & 68176 & 4109382 & 1.66 \\
\hline 19 & $05.07-11.07$ & 35178 & 1464504 & 2.40 & 39 & $22.11-28.11$ & 71707 & 4072184 & 1.76 \\
\hline 20 & $12.07-18.07$ & 36943 & 1581194 & 2.34 & 40 & 29.11-05.12 & 75697 & 4256119 & 1.78 \\
\hline
\end{tabular}

* Mortality rate was calculated as the ratio between the number of "Weekly Deaths" and the number of "Weekly New Cases"; it is expressed in percentage.

Conclusion: There are no valid publications that have revealed change in the danger of a new virus. Therefore, one can assume that the dynamic of the mortality rate among patients with CoViD-19 (Fig. 1) depended on the treatment efficacy. Based on the previous estimation offered by a group of experts from Imperial College London ${ }^{7}$ it may be concluded that a real amount of the infected people much higher than recorded and a real mortality rate is lower than was estimated in Tab. 2.

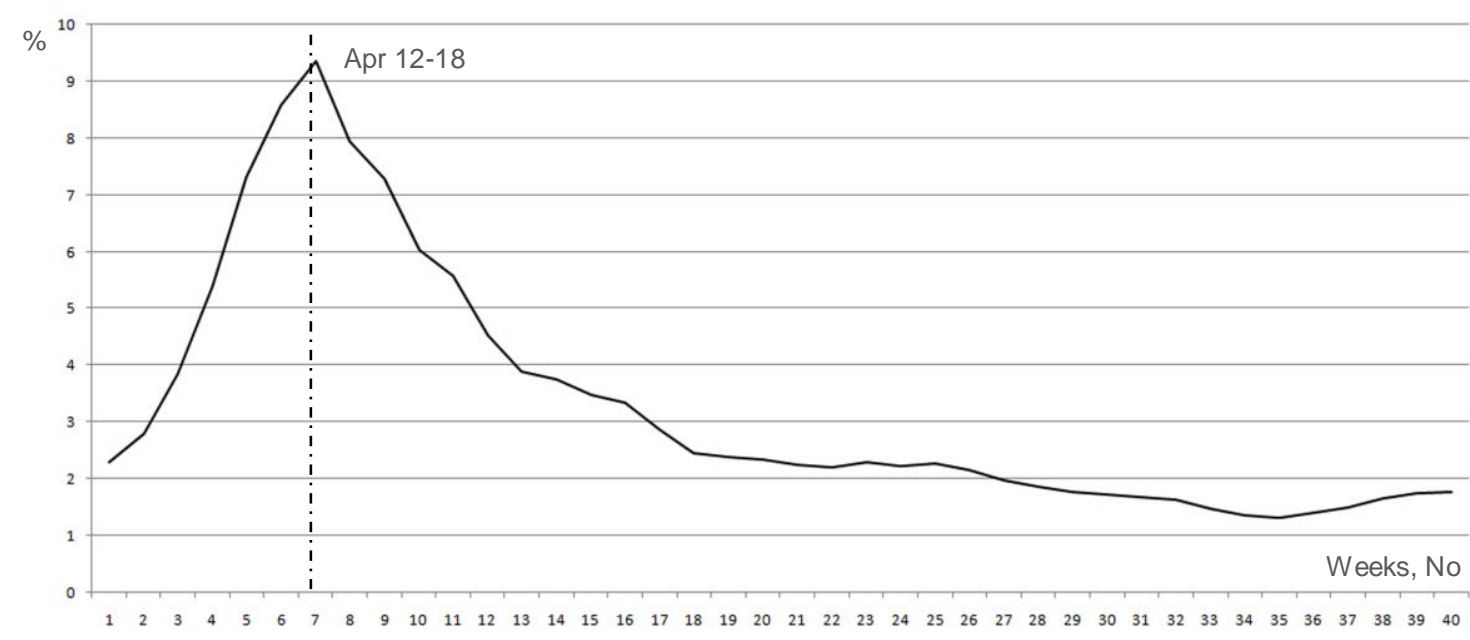

Figure 1. Weekly mortality rate calculated as a ratio between the total sum of weekly deaths due to CoViD-19 and the total sum of the weekly new cases infected with SARS-CoV-2 (week numbers see in the Tab. 2). 


\section{A Global Weekly Mortality Cycle Related to CoViD-19.}

Background: On the CoViD-19 mortality statistics presented on the 'Worldometer' website, ${ }^{12}$ a weekly cycle of decreased mortality on Sundays and Mondays is evident (Fig. 2). This cycle can vary in different countries, ${ }^{13}$ but the main trend is decreased mortality towards weekends. ${ }^{14}$

Objective: To study the weekly mortality cycle among CoViD-19 patients.

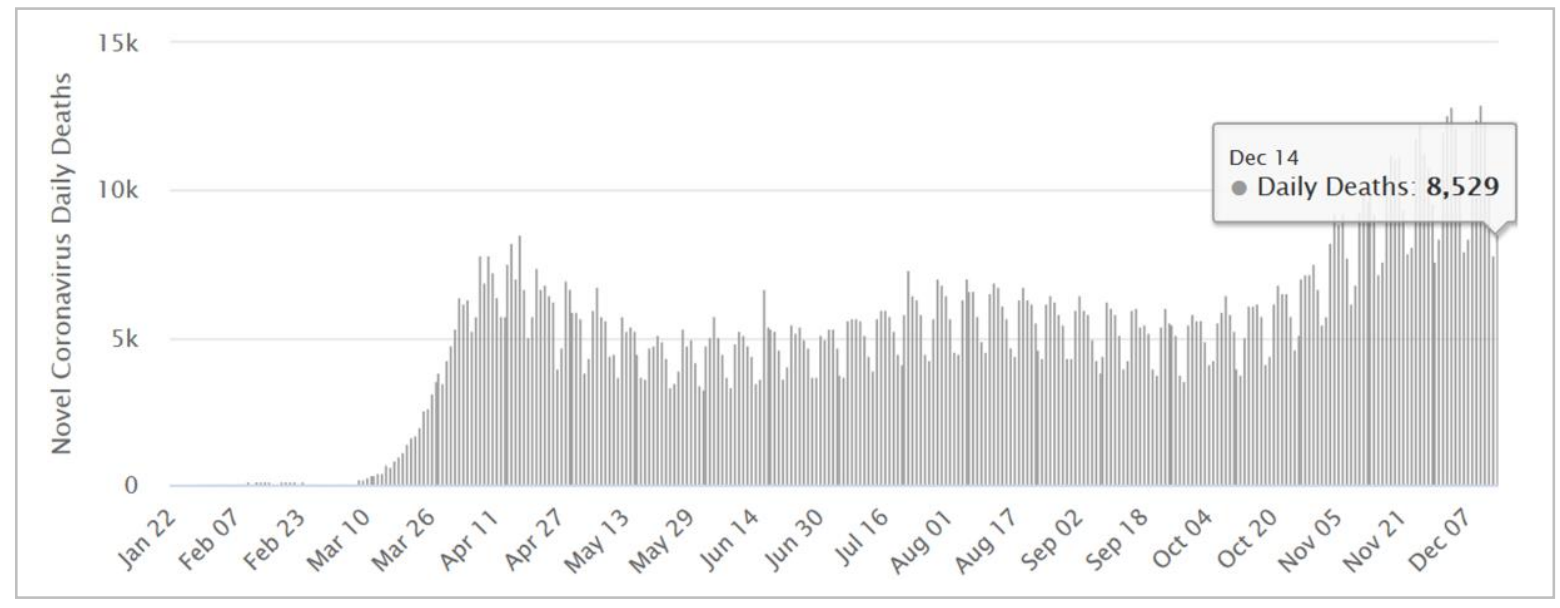

Figure 2. Daily New Deaths due to CoViD-19 worldwide (a screenshot taken on Dec. 15, 2020).

2.1. Methods: Since July 6, 2020, data from 'Worldometer' website were collected every 5-6 days in the form of numbers and screenshots. Data processing was divided into two parts: the first was calculating mortality on certain days of the week worldwide; the second was calculating mortality on certain days of the week in various countries.

Database of worldwide daily mortality due to CoViD-19 from January 24 to December 5, 2020, were collected on December 7, 2020 (Tab. 1).

There were comparisons between the total mortality on certain days of the week in absolute values and percentages. There was an additional criteria used, that was a ratio between a number of deaths on the day with the highest mortality and a number of deaths on the day with the lowest mortality, that can be termed as high/low mortality ratio or HLM. 40 weeks were analysed (01.03.20- 05.12.20).

Results: During the mentioned weeks there have been a total of 1,530,859 deaths from CoViD-19, worldwide. 166,688 (10.89\%) patients died on Sundays, 170,185 (11.12\%) on Mondays, 236,746 (15.46\%) on Tuesdays, 251,387 (16.42\%) on Wednesdays, 246,224 (16.08\%) on Thursdays, 242,776 (15.86\%) on Fridays and 216,853 (14.17\%) on Saturdays.

The highest daily mortality was on Wednesday and the lowest daily mortality was on Sunday. The HLM ratio, calculated as 251,387 divided by 166,688 was 1.508 (Tab 3). 
Table 3.

Absolute Values of deaths from CoViD-19 and Percentage in total and on certain days of the week.

Forty weeks were analysed (01.03.20-05.12.20). Database was collected on December 7, 2020.

\begin{tabular}{|l|r|r|r|r|r|r|r|r|r|}
\hline week, \# & Total & Sun & Mon & Tue & Wed & Thu & Fri & Sat & HLR \\
\hline Total, $\mathrm{n}$ & $1,530,859$ & 166,688 & 170,185 & 236,746 & 251,387 & 246,224 & 242,776 & 216,853 & 1.508 \\
\hline Mean & & 4,167 & 4,255 & 5,919 & 6,285 & 6,156 & 6,069 & 5,421 & \\
\hline SD \pm & & $\pm 1,600$ & $\pm 1,742$ & $\pm 2,474$ & $\pm 2,581$ & $\pm 2,452$ & $\pm 2,414$ & $\pm 2,024$ & \\
\hline$\%$ & $100 \%$ & $10.89 \%$ & $11.12 \%$ & $15.46 \%$ & $16.42 \%$ & $16.08 \%$ & $15.86 \%$ & $14.17 \%$ & \\
\hline
\end{tabular}

1.2. Methods: The comparison of the global daily mortality between various days of the week done with the calculation of the $p$-value. If $p$-value was 0.001 or less, for example, 0.0005 , or 0.00005 , it was presented as $\mathrm{p}<0.001 .40$ weeks were analysed (01.03.20- 05.12.20).

Results: The global daily mortality on Sunday was less than global daily mortality on Tue, Wed, Thu, Fri $(\mathrm{p}<0.001)$ and Sat $(\mathrm{p}<0.005)$. The global daily mortality on Monday was less than global daily mortality on Tue, Wed, Thu, Fri $(\mathrm{p}<0.001)$ and Sat $(\mathrm{p}<0.01)$.

Discussion: If the low mortality on Sundays was related to the registration of the deceased, one could assume that all cases not recorded on Sundays must be added to the death cases on Mondays, and as a result, the number of deaths on Mondays must be not less than on other days of the week. In reality, the number of deaths on Mondays was almost as low as on Sundays.

Conclusion: For patients suffering from CoViD-19 worldwide, the most favorable or safest days of the week were Sundays and Mondays (Fig. 3).

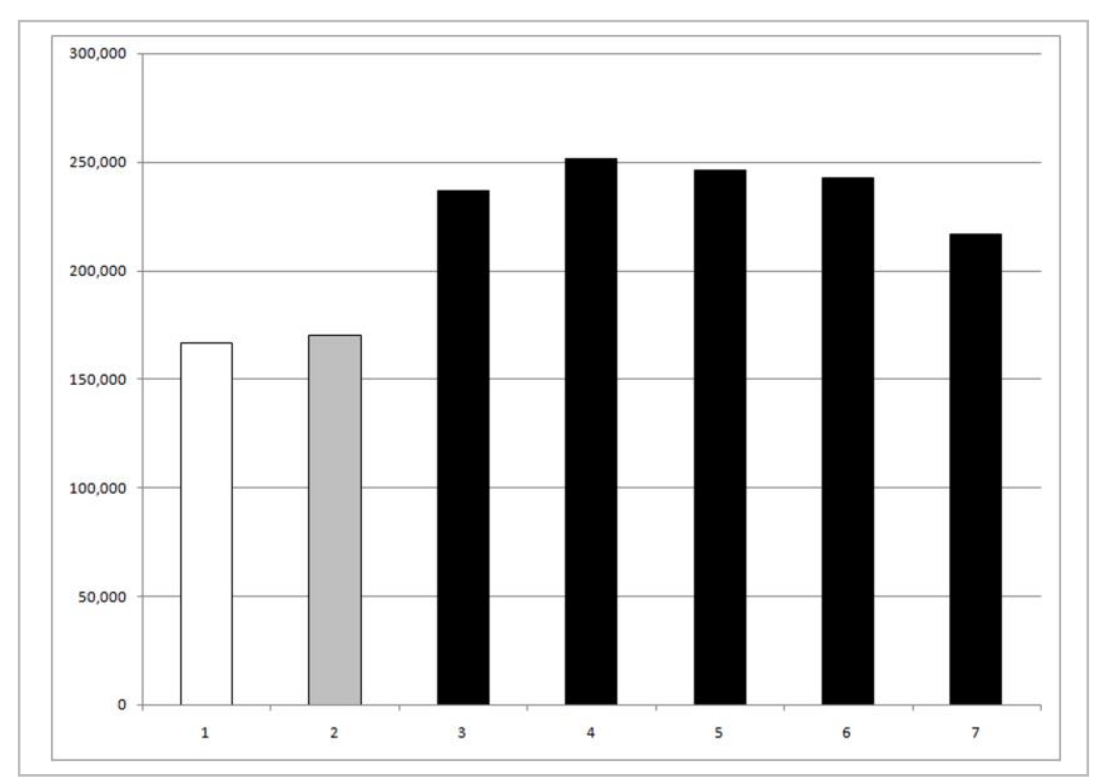

Figure 3. Global Mortality Due to CoViD-19 on Various Days of the Week (01.03.20 - 05.12.20). A vertical axis shows absolute value of deaths; a horizontal axis shows day of the week: 1. Sunday (white), 2. Monday (grey), 3. Tuesday, 4. Wednesday, 5. Thursday, 6. Friday, 7. Saturday (all black). 


\section{The Weekly Mortality Cycle related to CoViD-19 in Various Countries.}

Background: The global trend that may be termed the "Weekly Mortality Cycle" with more or less certainty, has been revealed in twelve countries including Argentina, Brazil, Chile, France, Germany, Mexico, the Netherlands, Poland, Russia, Ukraine, the United Kingdom and the United States.

As it has been presented above, the lowest worldwide mortality due to CoViD-19 was on Sundays and Mondays. In various countries days of the week with the lowest mortality can vary nevertheless they either belong to the weekend or they are near the weekend.

Due to the fact that the time of CoViD-19 outbreak varied in the different countries, the analysed weeks varied too. All analysed periods started on a Sunday and ended on a Saturday.

3.1. Methods: The data on mortality from CoViD-19 in different countries were taken from the website of 'Worldometer'. ${ }^{13}$ A comparison of the total mortality on certain days of the week in absolute values, percentages and HLM ratio in various countries was carried out (Table 4).

Table 4:

Mortality on various days of the week. There are absolute values, percentages and HLM ratio. Database was collected on December 9, 2020.

\begin{tabular}{|c|c|c|c|c|c|c|c|c|c|}
\hline $\begin{array}{l}\text { Countries: } \\
\text { Dates: }\end{array}$ & Total & Sun & Mon & Tue & Wed & Thu & Fri & Sat & HLM \\
\hline $\begin{array}{l}\text { United States } \\
22.03-05.12 .20\end{array}$ & 287,469 & $\begin{array}{r}* \mathbf{2 2 , 6 4 2} \\
7.87 \%\end{array}$ & $\begin{array}{l}26,410 \\
9.19 \%\end{array}$ & $\begin{array}{r}50,763 \\
17.66 \%\end{array}$ & $\begin{array}{r}52,624 \\
18.31 \%\end{array}$ & $\begin{array}{r}48,746 \\
16.96 \%\end{array}$ & $\begin{array}{r}46,973 \\
16.34 \%\end{array}$ & $\begin{array}{r}39,311 \\
13.67 \%\end{array}$ & 2.324 \\
\hline $\begin{array}{l}\text { Brazil } \\
\quad 22.03-05.12 .20\end{array}$ & 176,623 & $\begin{array}{l}\mathbf{1 3 , 6 7 3} \\
7.74 \%\end{array}$ & $\begin{array}{l}17,008 \\
9.63 \%\end{array}$ & $\begin{array}{r}30,389 \\
17.21 \%\end{array}$ & $\begin{array}{r}31,400 \\
17.78 \%\end{array}$ & $\begin{array}{r}31,477 \\
17.82 \%\end{array}$ & $\begin{array}{r}28,211 \\
16.00 \%\end{array}$ & $\begin{array}{r}24,465 \\
13.85 \%\end{array}$ & 2.302 \\
\hline $\begin{array}{l}\text { Mexico } \\
05.04-05.12 .20\end{array}$ & 108,840 & $\begin{array}{r}15,062 \\
13.84 \\
\end{array}$ & $\begin{array}{r}7,945 \\
7.3 \\
\end{array}$ & $\begin{array}{r}9,576 \\
8.8 \\
\end{array}$ & $\begin{array}{r}20,641 \\
18.96 \\
\end{array}$ & $\begin{array}{r}19,820 \\
18.21 \\
\end{array}$ & $\begin{array}{r}17,802 \\
16.36 \\
\end{array}$ & $\begin{array}{r}17,994 \\
16.53 \\
\end{array}$ & 2.598 \\
\hline $\begin{array}{l}\text { United Kingdom } \\
22.03-05.12 .20\end{array}$ & 60,746 & $\begin{array}{r}5,235 \\
8.62 \% \\
\end{array}$ & $\begin{array}{r}\mathbf{4 , 8 4 7} \\
7.98 \% \\
\end{array}$ & $\begin{array}{r}11,121 \\
18.31 \% \\
\end{array}$ & $\begin{array}{r}10,803 \\
17.78 \% \\
\end{array}$ & $\begin{array}{r}9,611 \\
15.82 \% \\
\end{array}$ & $\begin{array}{r}10,172 \\
16.75 \% \\
\end{array}$ & $\begin{array}{r}8,957 \\
14.74 \% \\
\end{array}$ & 2.294 \\
\hline $\begin{array}{l}\text { France } \\
\quad 22.03-05.12 .20\end{array}$ & 54,312 & $\begin{array}{r}\mathbf{4 , 5 7 2} \\
8.42 \% \\
\end{array}$ & $\begin{array}{r}7,690 \\
14.16 \% \\
\end{array}$ & $\begin{array}{r}9,907 \\
18.24 \% \\
\end{array}$ & $\begin{array}{r}7,734 \\
14.24 \% \\
\end{array}$ & $\begin{array}{r}8,784 \\
16.17 \% \\
\end{array}$ & $\begin{array}{r}9,572 \\
17.63 \% \\
\end{array}$ & $\begin{array}{r}6,053 \\
11.14 \% \\
\end{array}$ & 2.167 \\
\hline $\begin{array}{l}\text { Russia } \\
\quad 29.03-05.12 .20 \\
\end{array}$ & 42,620 & $\begin{array}{r}4,614 \\
10.83 \% \\
\end{array}$ & $\begin{array}{r}\mathbf{4 , 2 3 6} \\
9.94 \% \\
\end{array}$ & $\begin{array}{r}6,672 \\
15.66 \% \\
\end{array}$ & $\begin{array}{r}6,994 \\
16.41 \% \\
\end{array}$ & $\begin{array}{r}6,641 \\
15.58 \% \\
\end{array}$ & $\begin{array}{r}6,817 \\
15.99 \% \\
\end{array}$ & $\begin{array}{r}6,646 \\
15.59 \% \\
\end{array}$ & 1.651 \\
\hline $\begin{array}{l}\text { Argentina } \\
\quad 29.03-05.12 .20\end{array}$ & 39,578 & $\begin{array}{r}\mathbf{3 , 8 6 7} \\
9.77 \% \\
\end{array}$ & $\begin{array}{r}6,009 \\
15.18 \% \\
\end{array}$ & $\begin{array}{r}6,201 \\
15.67 \% \\
\end{array}$ & $\begin{array}{r}6,275 \\
15.86 \% \\
\end{array}$ & $\begin{array}{r}5,977 \\
15.10 \% \\
\end{array}$ & $\begin{array}{r}6,447 \\
16.29 \% \\
\end{array}$ & $\begin{array}{r}4,802 \\
12.13 \% \\
\end{array}$ & 1.667 \\
\hline $\begin{array}{l}\text { Poland } \\
\quad 29.03-05.12 .20\end{array}$ & 19,841 & $\begin{array}{r}1,719 \\
8.66 \%\end{array}$ & $\begin{array}{r}\mathbf{1 , 0 8 3} \\
5.46 \%\end{array}$ & $\begin{array}{r}2,708 \\
13.65 \%\end{array}$ & $\begin{array}{r}3,709 \\
18.69 \%\end{array}$ & $\begin{array}{r}3,557 \\
17.93 \%\end{array}$ & $\begin{array}{r}3,532 \\
17.80 \%\end{array}$ & $\begin{array}{r}3,533 \\
17.81 \%\end{array}$ & 3.425 \\
\hline $\begin{array}{l}\text { Germany } \\
22.03-05.12 .20\end{array}$ & 18,955 & $\begin{array}{r}\mathbf{1 , 2 7 0} \\
6.70 \% \\
\end{array}$ & $\begin{array}{r}2,521 \\
13.30 \% \\
\end{array}$ & $\begin{array}{r}3,470 \\
18.31 \% \\
\end{array}$ & $\begin{array}{r}3,485 \\
18.39 \% \\
\end{array}$ & $\begin{array}{r}3,276 \\
17.28 \% \\
\end{array}$ & $\begin{array}{r}3,092 \\
16.31 \% \\
\end{array}$ & $\begin{array}{r}1,841 \\
9.71 \% \\
\end{array}$ & 2.744 \\
\hline $\begin{array}{l}\text { Chile } \\
\quad 05.04-05.12 .20 \\
\end{array}$ & 15,037 & $\begin{array}{r}2,313 \\
15.38 \% \\
\end{array}$ & $\begin{array}{r}1,753 \\
11.66 \% \\
\end{array}$ & $\begin{array}{r}\mathbf{1 , 1 0 5} \\
7.35 \% \\
\end{array}$ & $\begin{array}{r}1,885 \\
12.54 \% \\
\end{array}$ & $\begin{array}{r}2,930 \\
19.48 \% \\
\end{array}$ & $\begin{array}{r}2,507 \\
16.67 \% \\
\end{array}$ & $\begin{array}{r}2,544 \\
16.92 \% \\
\end{array}$ & 2.652 \\
\hline $\begin{array}{l}\text { Ukraine } \\
29.03-05.12 .20\end{array}$ & 13,412 & $\begin{array}{r}1,406 \\
10.48 \% \\
\end{array}$ & $\begin{array}{r}1,172 \\
8.74 \% \\
\end{array}$ & $\begin{array}{r}1,990 \\
14.84 \% \\
\end{array}$ & $\begin{array}{r}2,206 \\
16.45 \% \\
\end{array}$ & $\begin{array}{r}2,185 \\
16.29 \% \\
\end{array}$ & $\begin{array}{r}2,219 \\
16.54 \% \\
\end{array}$ & $\begin{array}{r}2,234 \\
16.66 \% \\
\end{array}$ & 1.906 \\
\hline $\begin{array}{l}\text { Netherlands } \\
22.03-05.12 .20\end{array}$ & 9,511 & $\begin{array}{r}903 \\
9.49 \% \\
\end{array}$ & $\begin{array}{r}\mathbf{7 7 3} \\
8.13 \% \\
\end{array}$ & $\begin{array}{r}1,691 \\
17.78 \%\end{array}$ & $\begin{array}{r}1,642 \\
17.27 \%\end{array}$ & $\begin{array}{r}1,560 \\
16.40 \%\end{array}$ & $\begin{array}{r}1,497 \\
15.74 \%\end{array}$ & $\begin{array}{r}1,445 \\
15.19 \%\end{array}$ & 2.188 \\
\hline
\end{tabular}

* Absolute values on the day with the lowest mortality are highlighted by using bold font. 
Results: A comparison of the total mortality on certain days of the week has revealed that in the analysed countries daily mortality on Sundays $(n=5)$ or Mondays $(n=6)$ was less than on other days of the week, excluding Chile, where the lowest mortality due to CoDiD-19 was on Tuesdays. The highest HLM ratio was in Poland (3.425), the lowest one was in Russia (1.651).

Conclusion: For patients suffering from CoViD-19, the safest days of the week were mostly Sundays or Mondays.

3.2. Background: There are several combinations of days with the lowest mortality, but the most common are Sundays and Mondays. These may be termed "Sunday Protective Phenomenon" (Fig. 4) and "Monday Protective Phenomenon" (Fig. 5) respectively.

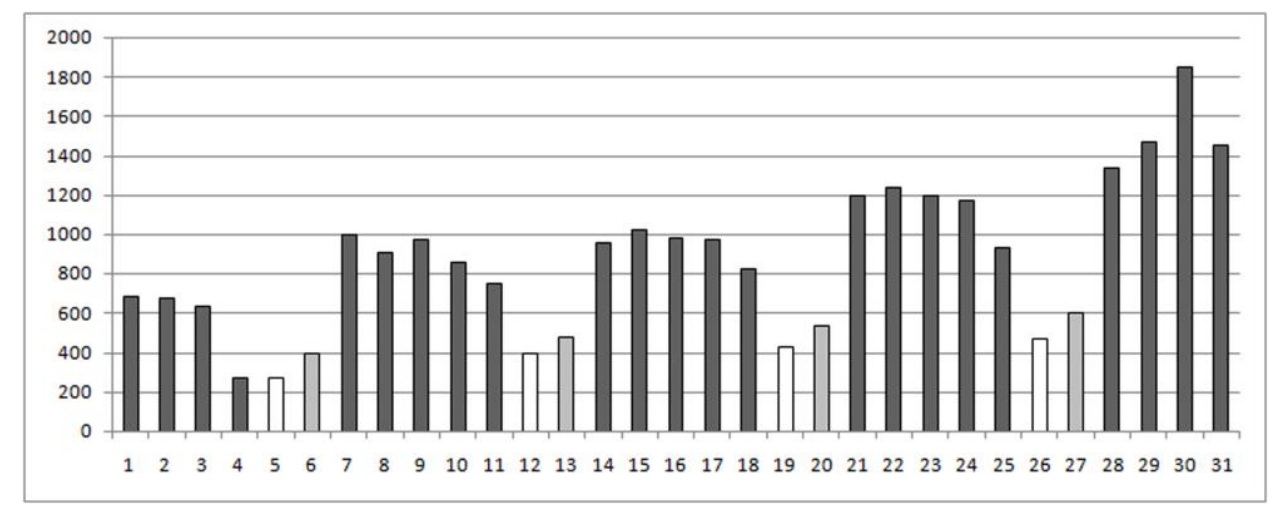

Figure 4. Daily New Deaths due to CoViD-19 in the United States, from July 1 to July 31, 2020.

A vertical axis shows absolute value of deaths; a horizontal axis shows day of the week:

Bars on the chart: Sunday is white color; Mondays is grey; Tuesdays, Wednesdays, Thursdays, Fridays and Saturdays in turn all are black. Database was collected on December 9, 2020.

In some countries analysis of the daily mortality revealed a stable weekly cycle with the one and the same day of the lowest mortality being either Sunday (Fig. 4) or Monday (Fig. 5); but in others, the day of the lowest mortality varied being both Sunday or Monday (Fig. 6).

3.2.1. "Sunday Protective Phenomenon" was discovered in Argentina, Brazil, France, Germany and the United States.

Methods: The comparison of the daily mortality between various days of the week in various countries done with the calculation of the $p$-value.

Argentina (29.03.20-05.12.20): On Sundays, the daily mortality was less than on Mon, Tue, Wed, Thu and Fri $(\mathrm{p}<0.05)$. 
Brazil (22.03.20-05.12.20): On Sundays, the daily mortality was less than on Tue, Wed, Thu, Fri and Sat $(\mathrm{p}<0.001)$. On Mondays, the daily mortality was less than on Tue, Wed, Thu, Fri $(\mathrm{p}<0.001)$ and Sat $(\mathrm{p}<0.005)$.

France (22.03.20-05.12.20): On Sundays, the daily mortality was less than on Tue and Fri $(\mathrm{p}<0.05)$.

Germany (22.03.20-05.12.20): On Sundays, the daily mortality was less than on Tue, Wed $(\mathrm{p}<0.01)$, Mon, Thu and Fri $(\mathrm{p}<0.05)$.

The United States of America (22.03.20-05.12.20): On Sundays, the daily mortality was less than on Tue, Wed, Thu, Fri and Sat ( $\mathrm{p}<0.001)$. On Mondays, the daily mortality was less than on Tue, Wed, Thu, Fri $(\mathrm{p}<0.001)$ and Sat $(\mathrm{p}<0.005)$.

3.2.2. "Monday Protective Phenomenon" discovered in Mexico, the Netherlands, Poland, Russia, Ukraine, the United Kingdom.

Methods: The comparison of the daily mortality between various days of the week in various countries done with the calculation of the $p$-value.

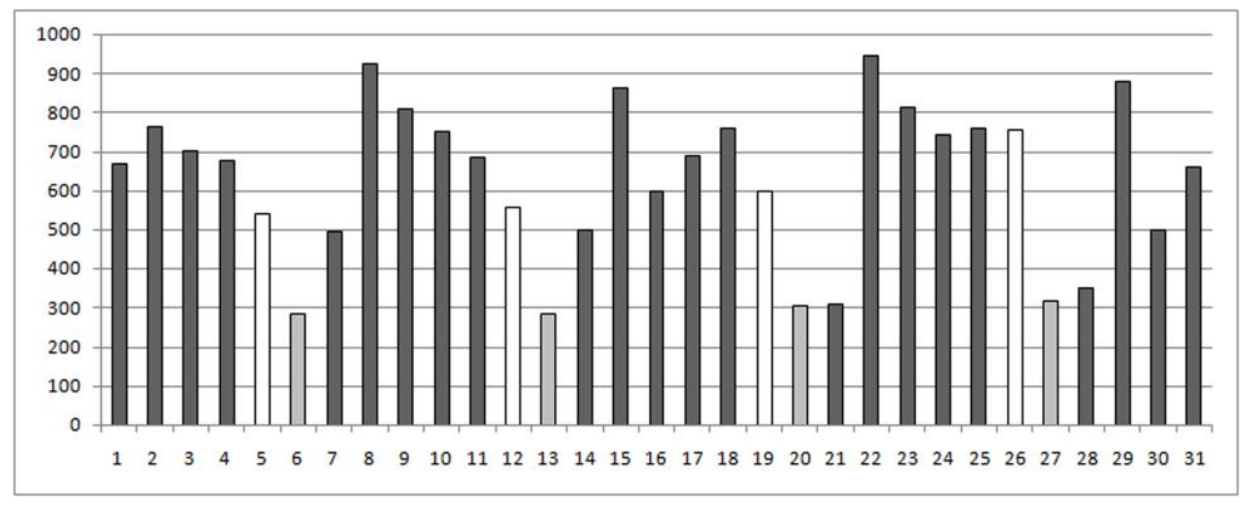

Figure 5. Daily New Death due to CoViD-19 in Mexico, from July 1 to July 31, 2020.

A vertical axis shows absolute value of deaths; a horizontal axis shows day of the week:

Bars on the chart: Sunday is white color; Mondays is grey; Tuesdays, Wednesdays, Thursdays, Fridays and Saturdays in turn all are black. Database was collected on December 9, 2020.

Mexico (05.04.20-05.12.20: On Mondays, the daily mortality was less than on Sun, Thu, Wed, Fri and Sat $(\mathrm{p}<0.001)$. On Tuesdays, the daily mortality was less than on Thu, Wed, Fri, Sat $(\mathrm{p}<0.001)$ and $\operatorname{Sun}(\mathrm{p}<0.005)$.

The Netherlands (22.03.20-05.12.20): On Mondays, the daily mortality was less than on Tue, Wed, Thu, Fri and Sat $(\mathrm{p}<0.05)$.

Poland (29.03.20-05.12.20): On Mondays, the daily mortality was less than on Wed, Thu, Fri and Sat $(\mathrm{p}<0.05)$. 
Russia (22.03.20-05.12.20): On Mondays, the daily mortality was less than on Wed, Fri ( $\mathrm{p}<0.01)$, Tue, Thu and Sat $(\mathrm{p}<0.05)$. On Sundays, the daily mortality was less than on Tue, Wed, Fri, Sat $(\mathrm{p}<0.05)$ and Thu $(\mathrm{p}<0.05240)$.

Ukraine (29.03.20-05.12.20): On Mondays, the daily mortality was less than on Wed, Thu, Fri and Sat $(\mathrm{p}<0.05)$.

The United Kingdom (22.03.20-05.12.20): On Mondays, the daily mortality was less than on Tue, Wed $(\mathrm{p}<0.01)$, Thu, Fri and Sat $(\mathrm{p}<0.05)$. On Sundays, the daily mortality was less than on Tue, Wed, Thu and Fri $(\mathrm{p}<0.05)$.

3.2.3. In Chile (05.04.20-05.12.20) the day of the week with the lowest mortality was Tuesday. On Tuesdays, the daily mortality was less than on Thu, Fri $(\mathrm{p}<0.001)$, Sun, Mon and Sat $(\mathrm{p}<0.005)$. On Mondays, the daily mortality was less than on Thu $(\mathrm{p}<0.005)$.

Conclusion: The weekly mortality cycle was discovered in twelve countries, including Argentina, Brazil, Chile, France, Germany, Mexico, the Netherlands, Poland, Russia, Ukraine, the United Kingdom and the United States of America. In the majority of these countries, for patients suffering from CoViD-19, the safest days of the week were either Sundays or Mondays. In Chile, the safest days of the week were Mondays and Tuesdays (Tab. 4).

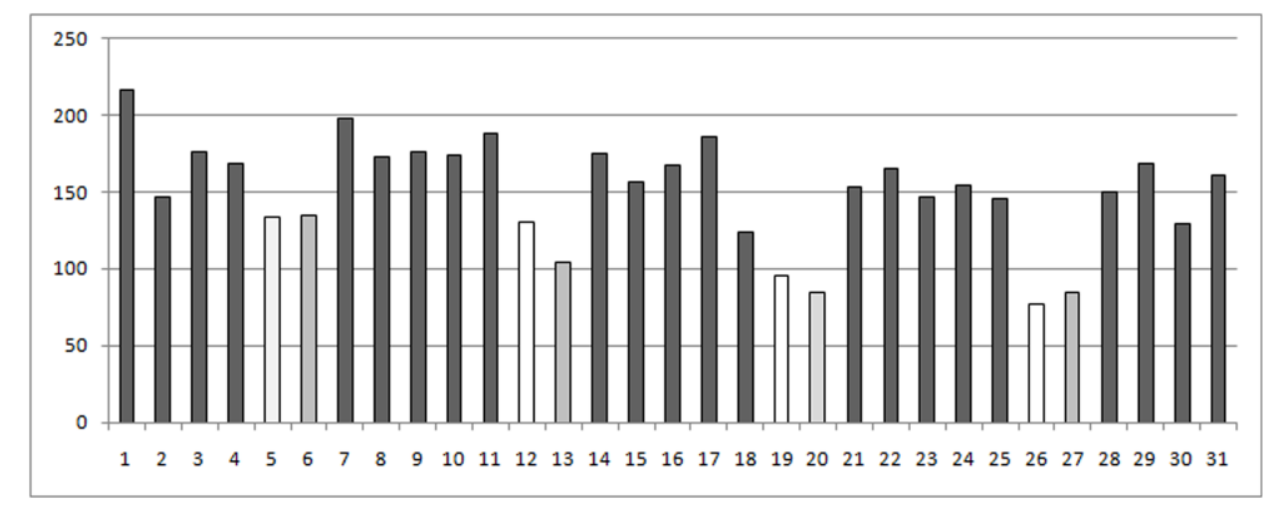

Figure 6. Daily New Deaths due to CoViD-19 in Russia, from July 1 to July 31, 2020. A vertical axis shows absolute value of deaths; a horizontal axis shows day of the week: Bars on the chart: Sunday is white color; Mondays is grey; Tuesdays, Wednesdays, Thursdays, Fridays and Saturdays in turn all are black. Database was collected on December 9, 2020.

\section{The Weekly Mortality Cycle in the States of the United States with the highest mortality.}

Background: Due to the fact that the United States had the highest numbers of deaths related to CoViD-19, ${ }^{15}$ the weekly mortality cycle was analysed in the twenty five States with the highest mortality ( 4000 cases and above). Database was collected on December 10-11, 2020. 
Table 5.

Total Mortality and Mortality on Various Days of the Week in the certain States of the United States.

There are absolute values, percentages and HLM ratio. Database was collected on December 10-11, 2020.

\begin{tabular}{|c|c|c|c|c|c|c|c|c|c|}
\hline $\begin{array}{l}\text { Countries: } \\
\\
\text { Dates: }\end{array}$ & Total, n & Sun & Mon & Tue & Wed & Thu & Fri & Sat & HLM \\
\hline $\begin{array}{l}\text { United States } \\
22.03-05.12 .20\end{array}$ & 287,469 & $\begin{array}{r}* 22,642 \\
7.87 \%\end{array}$ & $\begin{array}{l}26,410 \\
9.19 \%\end{array}$ & $\begin{array}{r}50,763 \\
17.66 \%\end{array}$ & $\begin{array}{r}52,624 \\
18.31 \%\end{array}$ & $\begin{array}{r}48,746 \\
16.96 \%\end{array}$ & $\begin{array}{r}46,973 \\
16.34 \%\end{array}$ & $\begin{array}{r}39,311 \\
13.67 \%\end{array}$ & 2.324 \\
\hline $\begin{array}{l}\text { 1. New York** } \\
22.03-05.12 .20\end{array}$ & 34,894 & $\begin{array}{r}\mathbf{4 , 7 5 9} \\
13.64 \%\end{array}$ & $\begin{array}{r}4,770 \\
13.67 \%\end{array}$ & $\begin{array}{r}5,528 \\
15.84 \%\end{array}$ & $\begin{array}{r}5,561 \\
15.94 \%\end{array}$ & $\begin{array}{r}4,691 \\
13.44 \%\end{array}$ & $\begin{array}{r}4,931 \\
14.13 \%\end{array}$ & $\begin{array}{r}4,654 \\
13.34 \%\end{array}$ & 1.689 \\
\hline $\begin{array}{l}\text { 2. Texas } \\
22.03-05.12 .20\end{array}$ & 23,174 & $\begin{array}{r}\mathbf{1 , 4 6 1} \\
6.30 \%\end{array}$ & $\begin{array}{r}1,781 \\
7.69 \%\end{array}$ & $\begin{array}{r}3,712 \\
16.02 \%\end{array}$ & $\begin{array}{r}4,327 \\
18.67 \% \\
\end{array}$ & $\begin{array}{r}4,768 \\
20.57 \%\end{array}$ & $\begin{array}{r}4,048 \\
17.47 \%\end{array}$ & $\begin{array}{r}3,077 \\
13.28 \%\end{array}$ & 3.264 \\
\hline $\begin{array}{l}\text { 3. California } \\
22.03-05.12 .20\end{array}$ & 19,855 & $\begin{array}{r}990 \\
4.99 \% \\
\end{array}$ & $\begin{array}{r}1,831 \\
9.22 \%\end{array}$ & $\begin{array}{r}3,667 \\
18.47 \%\end{array}$ & $\begin{array}{r}3,889 \\
19.59 \%\end{array}$ & $\begin{array}{r}3,441 \\
17.33 \%\end{array}$ & $\begin{array}{r}3,600 \\
18.13 \%\end{array}$ & $\begin{array}{r}2,437 \\
12.27 \%\end{array}$ & 3.928 \\
\hline $\begin{array}{l}\text { 4. Florida } \\
22.03-05.12 .20\end{array}$ & 19,072 & $\begin{array}{r}\mathbf{1 , 1 9 5} \\
6.27 \% \\
\end{array}$ & $\begin{array}{r}1,487 \\
7.80 \% \\
\end{array}$ & $\begin{array}{r}3,447 \\
18.07 \% \\
\end{array}$ & $\begin{array}{r}3,452 \\
18.10 \% \\
\end{array}$ & $\begin{array}{r}3,515 \\
18.43 \% \\
\end{array}$ & $\begin{array}{r}3,308 \\
17.34 \% \\
\end{array}$ & $\begin{array}{r}2,668 \\
13.99 \% \\
\end{array}$ & 2.941 \\
\hline $\begin{array}{l}\text { 5. New Jersey } \\
22.03-05.12 .20\end{array}$ & 17,412 & $\begin{array}{r}1,424 \\
8.18 \% \\
\end{array}$ & $\begin{array}{r}\mathbf{1 , 2 8 9} \\
7.40 \% \\
\end{array}$ & $\begin{array}{r}3,164 \\
18.17 \% \\
\end{array}$ & $\begin{array}{r}3,237 \\
18.59 \% \\
\end{array}$ & $\begin{array}{r}3,288 \\
18.88 \% \\
\end{array}$ & $\begin{array}{r}2,590 \\
14.88 \% \\
\end{array}$ & $\begin{array}{r}2,420 \\
13.90 \% \\
\end{array}$ & 2.551 \\
\hline $\begin{array}{l}\text { 6. Illinois } \\
\quad 22.03-05.12 .20\end{array}$ & 13,996 & $\begin{array}{r}1,074 \\
7.67 \%\end{array}$ & $\begin{array}{r}\mathbf{9 4 2} \\
6.73 \% \\
\end{array}$ & $\begin{array}{r}2,343 \\
16.74 \%\end{array}$ & $\begin{array}{r}2,763 \\
19.74 \%\end{array}$ & $\begin{array}{r}2,503 \\
17.89 \%\end{array}$ & $\begin{array}{r}2,182 \\
15.59 \%\end{array}$ & $\begin{array}{r}2,189 \\
15.64 \%\end{array}$ & 2.933 \\
\hline $\begin{array}{l}\text { 7. Pennsylvania } \\
22.03-05.12 .20\end{array}$ & 11,349 & $\begin{array}{r}\mathbf{6 4 7} \\
5.70 \%\end{array}$ & $\begin{array}{r}766 \\
6.75 \% \\
\end{array}$ & $\begin{array}{r}2,022 \\
17.82 \%\end{array}$ & $\begin{array}{r}2,436 \\
21.46 \%\end{array}$ & $\begin{array}{r}2,168 \\
19.10 \%\end{array}$ & $\begin{array}{r}1,844 \\
16.25 \%\end{array}$ & $\begin{array}{r}1,466 \\
12.92 \%\end{array}$ & 3.765 \\
\hline $\begin{array}{l}\text { 8. Massachusetts } \\
22.03-05.12 .20\end{array}$ & 10,962 & $\begin{array}{r}1,444 \\
13.17 \% \\
\end{array}$ & $\begin{array}{r}\mathbf{1 , 1 7 2} \\
10.69 \% \\
\end{array}$ & $\begin{array}{r}1,241 \\
11.32 \% \\
\end{array}$ & $\begin{array}{r}2,150 \\
19.62 \% \\
\end{array}$ & $\begin{array}{r}1,722 \\
15.71 \% \\
\end{array}$ & $\begin{array}{r}1,684 \\
15.36 \% \\
\end{array}$ & $\begin{array}{r}1,549 \\
14.13 \% \\
\end{array}$ & .834 \\
\hline $\begin{array}{l}\text { 9. Michigan } \\
22.03-05.12 .20\end{array}$ & 10,290 & $\begin{array}{r}\mathbf{4 9 4} \\
4.80 \% \\
\end{array}$ & $\begin{array}{r}1,107 \\
10.76 \%\end{array}$ & $\begin{array}{r}2,058 \\
20.00 \%\end{array}$ & $\begin{array}{r}1,381 \\
13.42 \%\end{array}$ & $\begin{array}{r}1,962 \\
19.07 \%\end{array}$ & $\begin{array}{r}1,380 \\
13.41 \%\end{array}$ & $\begin{array}{r}1,908 \\
18.54 \%\end{array}$ & 4.166 \\
\hline $\begin{array}{l}\text { 10. Georgia } \\
22.03-05.12 .20\end{array}$ & 9,686 & $\begin{array}{r}\mathbf{5 7 0} \\
5.88 \% \\
\end{array}$ & $\begin{array}{r}945 \\
9.76 \% \\
\end{array}$ & $\begin{array}{r}1,760 \\
18.17 \%\end{array}$ & $\begin{array}{r}1,703 \\
17.58 \%\end{array}$ & $\begin{array}{r}1,624 \\
16.77 \%\end{array}$ & $\begin{array}{r}1,702 \\
17.57 \%\end{array}$ & $\begin{array}{r}1,382 \\
14.27 \%\end{array}$ & .088 \\
\hline $\begin{array}{l}11 . \text { Ohio } \\
22.03-05.12 .20\end{array}$ & 6,953 & $\begin{array}{r}\mathbf{3 6 5} \\
5.25 \% \\
\end{array}$ & $\begin{array}{r}593 \\
8.53 \% \\
\end{array}$ & $\begin{array}{r}1,349 \\
19.40 \% \\
\end{array}$ & $\begin{array}{r}1,579 \\
22.71 \% \\
\end{array}$ & $\begin{array}{r}1,106 \\
15.91 \% \\
\end{array}$ & $\begin{array}{r}1,199 \\
17.24 \% \\
\end{array}$ & $\begin{array}{r}762 \\
10.96 \% \\
\end{array}$ & 4.326 \\
\hline $\begin{array}{l}\text { 12. Arizona } \\
22.03-05.12 .20\end{array}$ & 6,927 & $\begin{array}{r}386 \\
5.57 \% \\
\end{array}$ & $\begin{array}{r}\mathbf{1 2 0} \\
1.73 \%\end{array}$ & $\begin{array}{r}1,174 \\
16.95 \% \\
\end{array}$ & $\begin{array}{r}1,485 \\
21.44 \% \\
\end{array}$ & $\begin{array}{r}1,277 \\
18.44 \% \\
\end{array}$ & $\begin{array}{r}1,159 \\
16.73 \% \\
\end{array}$ & $\begin{array}{r}1,326 \\
19.14 \% \\
\end{array}$ & 12.38 \\
\hline $\begin{array}{l}\text { 13. Louisiana } \\
22.03-05.12 .20\end{array}$ & 6,527 & $\begin{array}{r}945 \\
14.48 \%\end{array}$ & $\begin{array}{r}686 \\
10.51 \% \\
\end{array}$ & $\begin{array}{r}1,111 \\
17.02 \%\end{array}$ & $\begin{array}{r}1,218 \\
18.66 \%\end{array}$ & $\begin{array}{r}984 \\
15.08 \%\end{array}$ & $\begin{array}{r}1,176 \\
18.02 \%\end{array}$ & $\begin{array}{r}\mathbf{4 0 7} \\
6.23 \% \\
\end{array}$ & .993 \\
\hline $\begin{array}{l}\text { 14. Indiana } \\
22.03-05.12 .20\end{array}$ & 6,150 & $\begin{array}{r}\mathbf{4 3 3} \\
7.04 \%\end{array}$ & $\begin{array}{r}527 \\
8.57 \% \\
\end{array}$ & $\begin{array}{r}1,330 \\
21.63 \%\end{array}$ & $\begin{array}{r}989 \\
16.08 \%\end{array}$ & $\begin{array}{r}954 \\
15.51 \%\end{array}$ & $\begin{array}{r}989 \\
16.08 \%\end{array}$ & $\begin{array}{r}928 \\
15.09 \%\end{array}$ & 3.072 \\
\hline $\begin{array}{l}\text { 15. North Carolina } \\
\text { 29.03-05.12.20 }\end{array}$ & 5,511 & $\begin{array}{r}\mathbf{3 3 4} \\
6.06 \%\end{array}$ & $\begin{array}{r}335 \\
6.08 \%\end{array}$ & $\begin{array}{r}1,104 \\
20.04 \%\end{array}$ & $\begin{array}{r}1,083 \\
19.65 \%\end{array}$ & $\begin{array}{r}975 \\
17.69 \% \\
\end{array}$ & $\begin{array}{r}937 \\
17.00 \%\end{array}$ & $\begin{array}{r}743 \\
13.48 \%\end{array}$ & .305 \\
\hline $\begin{array}{r}\text { 16. Connecticut** } \\
22.03-05.12 .20 \\
\end{array}$ & 5,141 & $\begin{array}{r}447 \\
8.69 \% \\
\end{array}$ & $\begin{array}{r}853 \\
16.59 \% \\
\end{array}$ & $\begin{array}{r}641 \\
12.47 \% \\
\end{array}$ & $\begin{array}{r}978 \\
19.02 \% \\
\end{array}$ & $\begin{array}{r}788 \\
15.33 \% \\
\end{array}$ & $\begin{array}{r}848 \\
16.50 \% \\
\end{array}$ & $\begin{array}{r}586 \\
11.40 \% \\
\end{array}$ & 2.188 \\
\hline $\begin{array}{l}17 . \text { Tennessee } \\
29.03-05.12 .20\end{array}$ & 4,898 & $\begin{array}{r}\mathbf{2 8 0} \\
5.72 \% \\
\end{array}$ & $\begin{array}{r}475 \\
9.70 \% \\
\end{array}$ & $\begin{array}{r}931 \\
19.01 \% \\
\end{array}$ & $\begin{array}{r}802 \\
16.37 \% \\
\end{array}$ & $\begin{array}{r}938 \\
19.15 \% \\
\end{array}$ & $\begin{array}{r}879 \\
17.94 \% \\
\end{array}$ & $\begin{array}{r}593 \\
12.11 \% \\
\end{array}$ & 3.350 \\
\hline $\begin{array}{l}\text { 18. Maryland } \\
22.03-05.12 .20\end{array}$ & 4,831 & $\begin{array}{r}466 \\
9.64 \% \\
\end{array}$ & $\begin{array}{r}515 \\
10.66 \% \\
\end{array}$ & $\begin{array}{r}869 \\
17.99 \% \\
\end{array}$ & $\begin{array}{r}768 \\
15.90 \% \\
\end{array}$ & $\begin{array}{r}734 \\
15.19 \% \\
\end{array}$ & $\begin{array}{r}712 \\
14.74 \% \\
\end{array}$ & $\begin{array}{r}767 \\
15.88 \% \\
\end{array}$ & 1.865 \\
\hline $\begin{array}{l}\text { 19. Missouri } \\
22.03-05.12 .20\end{array}$ & 4,628 & $\begin{array}{r}354 \\
7.65 \% \\
\end{array}$ & $\begin{array}{r}\mathbf{2 7 1} \\
5.86 \% \\
\end{array}$ & $\begin{array}{r}1,083 \\
23.40 \% \\
\end{array}$ & $\begin{array}{r}793 \\
17.14 \% \\
\end{array}$ & $\begin{array}{r}742 \\
16.03 \% \\
\end{array}$ & $\begin{array}{r}798 \\
17.24 \% \\
\end{array}$ & $\begin{array}{r}587 \\
12.68 \% \\
\end{array}$ & 3.996 \\
\hline $\begin{array}{l}\text { 20. South Carolina } \\
22.03-05.12 .20\end{array}$ & 4,512 & $\begin{array}{r}\mathbf{3 2 7} \\
7.25 \% \\
\end{array}$ & $\begin{array}{r}374 \\
8.29 \% \\
\end{array}$ & $\begin{array}{r}769 \\
17.04 \% \\
\end{array}$ & $\begin{array}{r}831 \\
18.42 \% \\
\end{array}$ & $\begin{array}{r}754 \\
16.71 \% \\
\end{array}$ & $\begin{array}{r}691 \\
15.31 \% \\
\end{array}$ & $\begin{array}{r}766 \\
16.98 \% \\
\end{array}$ & 2.541 \\
\hline $\begin{array}{l}\text { 21. Virginia } \\
22.03-05.12 .20\end{array}$ & 4,208 & $\begin{array}{r}\mathbf{2 8 6} \\
6.80 \% \\
\end{array}$ & $\begin{array}{r}302 \\
7.18 \% \\
\end{array}$ & $\begin{array}{r}818 \\
19.44 \%\end{array}$ & $\begin{array}{r}797 \\
18.94 \%\end{array}$ & $\begin{array}{r}721 \\
17.13 \%\end{array}$ & $\begin{array}{r}673 \\
15.99 \%\end{array}$ & $\begin{array}{r}611 \\
14.52 \%\end{array}$ & 2.860 \\
\hline $\begin{array}{l}\text { 22. Minnesota } \\
29.03-05.12 .20\end{array}$ & 3,974 & $\begin{array}{r}503 \\
12.66 \% \\
\end{array}$ & $\begin{array}{r}\mathbf{2 4 7} \\
6.21 \% \\
\end{array}$ & $\begin{array}{r}426 \\
10.72 \% \\
\end{array}$ & $\begin{array}{r}786 \\
19.78 \%\end{array}$ & $\begin{array}{r}744 \\
18.72 \% \\
\end{array}$ & $\begin{array}{r}688 \\
17.31 \%\end{array}$ & $\begin{array}{r}580 \\
14.60 \%\end{array}$ & 3.182 \\
\hline $\begin{array}{l}\text { 23. Mississippi } \\
29.03-05.12 .20\end{array}$ & 3,934 & $\begin{array}{r}305 \\
7.75 \%\end{array}$ & $\begin{array}{r}\mathbf{2 4 8} \\
6.30 \%\end{array}$ & $\begin{array}{r}932 \\
23.69 \%\end{array}$ & $\begin{array}{r}796 \\
20.24 \%\end{array}$ & $\begin{array}{r}547 \\
13.91 \%\end{array}$ & $\begin{array}{r}571 \\
14.51 \%\end{array}$ & $\begin{array}{r}535 \\
13.60 \%\end{array}$ & 3.758 \\
\hline $\begin{array}{l}\text { 24. Alabama } \\
29.03-05.12 .20\end{array}$ & 3,873 & $\begin{array}{r}\mathbf{1 4 2} \\
3.67 \% \\
\end{array}$ & $\begin{array}{r}251 \\
6.48 \% \\
\end{array}$ & $\begin{array}{r}730 \\
18.85 \% \\
\end{array}$ & $\begin{array}{r}896 \\
23.15 \% \\
\end{array}$ & $\begin{array}{r}707 \\
18.26 \% \\
\end{array}$ & $\begin{array}{r}671 \\
17.30 \% \\
\end{array}$ & $\begin{array}{r}476 \\
12.29 \% \\
\end{array}$ & .310 \\
\hline $\begin{array}{l}\text { 25. Wisconsin } \\
29.03-05.12 .20\end{array}$ & 3,690 & $\begin{array}{r}164 \\
4.44 \% \\
\end{array}$ & $\begin{array}{r}\mathbf{1 5 7} \\
4.26 \% \\
\end{array}$ & $\begin{array}{r}843 \\
22.85 \% \\
\end{array}$ & $\begin{array}{r}672 \\
18.21 \% \\
\end{array}$ & $\begin{array}{r}645 \\
17.48 \% \\
\end{array}$ & $\begin{array}{r}589 \\
15.96 \% \\
\end{array}$ & $\begin{array}{r}620 \\
16.80 \% \\
\end{array}$ & 5.369 \\
\hline
\end{tabular}

* Absolute values on the day with the lowest mortality are highlighted by using bold font.

** Weekly Mortality Cycle has not been revealed in these States; in other States mentioned in this table Weekly Mortality Cycle has been revealed and confirmed. 
Methods: There were comparisons between the total mortality on certain days of the week in absolute values, percentages and a calculation of HLM ratio (Table 5). Additionally a comparison of daily mortality between certain days of the week with the calculation of the $p$-value has been done.

Results: In the majority of analysed states, for patients suffering from CoViD-19, the safest days of the week were either Sundays ( $n=16)$ or Mondays $(n=8)$.

Significant differences in mortality due to CoViD-19 between various days of the week were revealed in the states of Alabama, Arizona, California, Florida, Georgia, Illinois, Indiana, Louisiana, Maryland, Massachusetts, Michigan, Minnesota, Mississippi, Missouri, New Jersey, North Carolina, Ohio, Pennsylvania, South Carolina, Tennessee, Texas, Virginia and Wisconsin $(\mathrm{p}<0.05 \div \mathrm{p}<0.0001)$.

The highest HLM ratio was in Arizona (12.375), the lowest HLM ratio was in New York (1.689).

In the State of Louisiana (22.03.20-05.12.20), twenty three Saturdays out of thirty seven had no even one fatal case due to covid-19.

Conclusion: The weekly mortality cycle was discovered in the United States as a whole country and in the certain states. The safest days of the week were either Sundays or Mondays.

\section{Countries with the Unconfirmed Weekly Mortality Cycle.}

In Bangladesh, Belgium, Canada, Czechia, Colombia, India, Indonesia, Iran, Italy, Peru, Romania, Spain, South Africa, Sweden and some other countries, the difference in total mortality between certain days of the week was not significant ( $\mathrm{p}>0.05)$. Nevertheless, in the majority of countries examined, the days with the lowest mortality were either Sundays (Tab. 6) or Mondays (Tab. 7).

Table 6. Countries where Daily Mortality due to COVID-19 was the lowest on Sundays.

Mortality in various days of the week: there are absolute values, percentages and HLM ratio.

Database was collected on December 9, 2020.

\begin{tabular}{|c|c|c|c|c|c|c|c|c|c|}
\hline Country: & Total & Sun & Mon & Tue & Wed & Thu & Fri & Sat & HLM \\
\hline $\begin{array}{l}\text { Italy } \\
\quad 01.03-05.12 .20\end{array}$ & 59,482 & $\begin{array}{r}* \mathbf{6 , 8 5 6} \\
11.53 \%\end{array}$ & $\begin{array}{r}7,410 \\
12.46 \%\end{array}$ & $\begin{array}{r}8,954 \\
15.05 \%\end{array}$ & $\begin{array}{r}8,775 \\
14.75 \%\end{array}$ & $\begin{array}{r}9,197 \\
15.46 \%\end{array}$ & $\begin{array}{r}9,230 \\
15.52 \%\end{array}$ & $\begin{array}{r}9,060 \\
15.23 \%\end{array}$ & 1.346 \\
\hline $\begin{array}{l}\text { Spain } \\
08.03-05.12 .20\end{array}$ & 46,443 & $\begin{array}{r}\mathbf{5 , 2 5 4} \\
11.31 \%\end{array}$ & $\begin{array}{r}5,446 \\
11.73 \%\end{array}$ & $\begin{array}{r}7,980 \\
17.18 \%\end{array}$ & $\begin{array}{r}7,681 \\
16.54 \%\end{array}$ & $\begin{array}{r}7,149 \\
15.39 \%\end{array}$ & $\begin{array}{r}7,297 \\
15.71 \%\end{array}$ & $\begin{array}{r}5,636 \\
12.14 \%\end{array}$ & 1.519 \\
\hline $\begin{array}{l}\text { South Africa } \\
03.05-05.12 .20\end{array}$ & 21,561 & $\begin{array}{r}\mathbf{2 , 1 3 5} \\
9.90 \%\end{array}$ & $\begin{array}{r}2,675 \\
12.41 \%\end{array}$ & $\begin{array}{r}3,692 \\
17.12 \%\end{array}$ & $\begin{array}{r}3,315 \\
15.38 \%\end{array}$ & $\begin{array}{r}3,620 \\
16.79 \%\end{array}$ & $\begin{array}{r}3,302 \\
15.31 \%\end{array}$ & $\begin{array}{r}2,822 \\
13.09 \%\end{array}$ & 1.729 \\
\hline $\begin{array}{l}\text { Canada } \\
22.03-05.12 .20\end{array}$ & 12,455 & $\begin{array}{r}\mathbf{1 , 3 3 3} \\
10.70 \%\end{array}$ & $\begin{array}{r}1,502 \\
12.06 \%\end{array}$ & $\begin{array}{r}1,838 \\
14.76 \%\end{array}$ & $\begin{array}{r}1,879 \\
15.09 \%\end{array}$ & $\begin{array}{r}2,169 \\
17.41 \%\end{array}$ & $\begin{array}{r}1,872 \\
15.03 \%\end{array}$ & $\begin{array}{r}1,862 \\
14.95 \%\end{array}$ & 1.627 \\
\hline $\begin{array}{l}\text { Romania } \\
\quad 22.03-05.12 .20\end{array}$ & 12,186 & $\begin{array}{r}\mathbf{1 , 4 4 2} \\
11.83 \% \\
\end{array}$ & $\begin{array}{r}1,475 \\
12.11 \%\end{array}$ & $\begin{array}{r}1,991 \\
16.34 \%\end{array}$ & $\begin{array}{r}1,883 \\
15.45 \% \\
\end{array}$ & $\begin{array}{r}1,815 \\
14.89 \% \\
\end{array}$ & $\begin{array}{r}1,873 \\
15.37 \% \\
\end{array}$ & $\begin{array}{r}1,707 \\
14.01 \% \\
\end{array}$ & 1.381 \\
\hline $\begin{array}{l}\text { Czechia } \\
29.03-05.12 .20\end{array}$ & 8,802 & $\begin{array}{r}\mathbf{1 , 0 9 0} \\
12.38 \%\end{array}$ & $\begin{array}{r}1,274 \\
14.48 \%\end{array}$ & $\begin{array}{r}1,368 \\
15.54 \%\end{array}$ & $\begin{array}{r}1,360 \\
15.45 \%\end{array}$ & $\begin{array}{r}1,239 \\
14.08 \%\end{array}$ & $\begin{array}{r}1,381 \\
15.69 \%\end{array}$ & $\begin{array}{r}1,090 \\
12.38 \%\end{array}$ & 1.267 \\
\hline
\end{tabular}

* Absolute values on the day with the lowest mortality are highlighted by using bold font. 
Table 7. Countries where Daily Mortality due to COVID-19 was the lowest on Mondays. Mortality in various days of the week: there are absolute values, percentages and HLM ratio.

Database was collected on December 9, 2020.

\begin{tabular}{|c|c|c|c|c|c|c|c|c|c|}
\hline Country: & Total & Sun & Mon & Tue & Wed & Thu & Fri & Sat & HLM \\
\hline India & 138,456 & 18,562 & 17,829 & 19,965 & 20,814 & 20,760 & 20,636 & 19,890 & 1.167 \\
\hline 05.04-05.12.20 & & $13.41 \%$ & $12.88 \%$ & $14.42 \%$ & $15.03 \%$ & $14.99 \%$ & $14.90 \%$ & $14.37 \%$ & \\
\hline Colombia & 37,626 & 5,330 & 4,985 & 5,212 & 5,414 & 5,443 & 5,732 & 5,510 & 1.150 \\
\hline 29. & & $14.17 \%$ & $13.25 \%$ & $13.85 \%$ & $14.39 \%$ & $14.47 \%$ & $15.23 \%$ & $14.64 \%$ & \\
\hline Peru & 36,146 & 5,415 & 5,032 & 5,086 & 5,066 & 5,041 & 5,280 & 5,226 & 1.083 \\
\hline 22.0 & & $14.98 \%$ & $13.92 \%$ & $14.07 \%$ & $14.01 \%$ & $13.95 \%$ & $14.61 \%$ & $14.46 \%$ & \\
\hline Indone & 17,557 & 2,338 & 2,330 & 2,535 & 2,674 & 2,719 & 2,548 & 2,413 & 1.167 \\
\hline $22.03-0$ & & $13.32 \%$ & $13.27 \%$ & $14.44 \%$ & $15.23 \%$ & $15.49 \%$ & $14.51 \%$ & $13.74 \%$ & \\
\hline Belgium & 17,129 & 2,385 & 2,073 & 2,321 & 2,840 & 2,643 & 2,388 & 2,479 & 1.370 \\
\hline $15.03-05.12 .20$ & & $13.93 \%$ & $12.10 \%$ & $13.55 \%$ & $16.58 \%$ & $15.43 \%$ & $13.94 \%$ & $14.47 \%$ & \\
\hline
\end{tabular}

* Absolute values on the day with the lowest mortality are highlighted by using bold font.

Table 8. Countries where Daily Mortality due to COVID-19 was the lowest on Tuesdays, Fridays or Saturdays. Mortality in various days of the week: there are absolute values, percentages and HLM ratio.

Database was collected on December 9, 2020.

\begin{tabular}{|c|c|c|c|c|c|c|c|c|c|}
\hline Country: & Total & Sun & Mon & Tue & Wed & Thu & Fri & Sat & HLM \\
\hline $\begin{array}{l}\text { Iran } \\
\quad 01.03-05.12 .20\end{array}$ & 49,973 & $\begin{array}{l}6,965 \\
13.94\end{array}$ & $\begin{array}{l}7,261 \\
14.53\end{array}$ & $\begin{array}{l}7,265 \\
14.54\end{array}$ & $\begin{array}{l}7,335 \\
14.68\end{array}$ & $\begin{array}{l}7,323 \\
14.65\end{array}$ & $\begin{array}{l}6,969 \\
13.94\end{array}$ & $\begin{array}{l}\mathbf{6 , 8 5 5} \\
13.72\end{array}$ & 1.070 \\
\hline $\begin{array}{l}\text { Sweden } \\
15.03-05.12 .20\end{array}$ & 7,137 & $\begin{array}{l}1,022 \\
14.32\end{array}$ & $\begin{array}{l}1,041 \\
14.59\end{array}$ & $\begin{array}{r}986 \\
13.81\end{array}$ & $\begin{array}{l}1,055 \\
14.78\end{array}$ & $\begin{array}{l}1,037 \\
14.53\end{array}$ & $\begin{array}{l}1,004 \\
14.07\end{array}$ & $\begin{array}{r}992 \\
13.9\end{array}$ & 1.070 \\
\hline $\begin{array}{l}\text { Bangladesh } \\
05.04-05.12 .20\end{array}$ & 6,805 & $\begin{array}{r}957 \\
14.06\end{array}$ & $\begin{array}{r}982 \\
14.43\end{array}$ & $\begin{array}{r}1,055 \\
15.5\end{array}$ & $\begin{array}{l}1,008 \\
14.82\end{array}$ & $\begin{array}{r}995 \\
14.62\end{array}$ & $\begin{array}{r}898 \\
13.2\end{array}$ & $\begin{array}{r}910 \\
13.37\end{array}$ & 1.174 \\
\hline
\end{tabular}

\section{Remarks:}

The highest HLM ratio was in South Africa (1.729), the lowest one was in Iran and Sweden (1.070).

The first patients with CoViD-19 were identified in China, ${ }^{16}$ but the local epidemic ended in March, 2020; next waves of the epidemic were not reported (Fig. 7).

\section{Revision of some numbers dealing with mortality due to CoViD-19.}

In several countries after revision of death records related to CoViD-19, some amount of the fatal cases were added, and this fact can be recognized as a sharp spike of deaths in the daily mortality chart.

Sudden increase of deaths number happened in China on April 17, 2020, when 1,290 new fatal cases were added (Fig. 6). ${ }^{16}$ The local authorities provided the following explanation: "The revisions were made in accordance with related laws and regulations, as well as the principle of being responsible for history, the people and the deceased". ${ }^{17}$

Similar corrections happened in Canada on May 31, 2020, when 222 new fatal cases were added, ${ }^{18}$ in Chile on June 7, 2020, when new 653 fatal cases were added, ${ }^{19}$ and in India on June 16, 2020, when new 2006 fatal cases were added. ${ }^{20}$ In all these cases to calculate weekly mortality cycle an average daily mortality of the affected week was applied. 


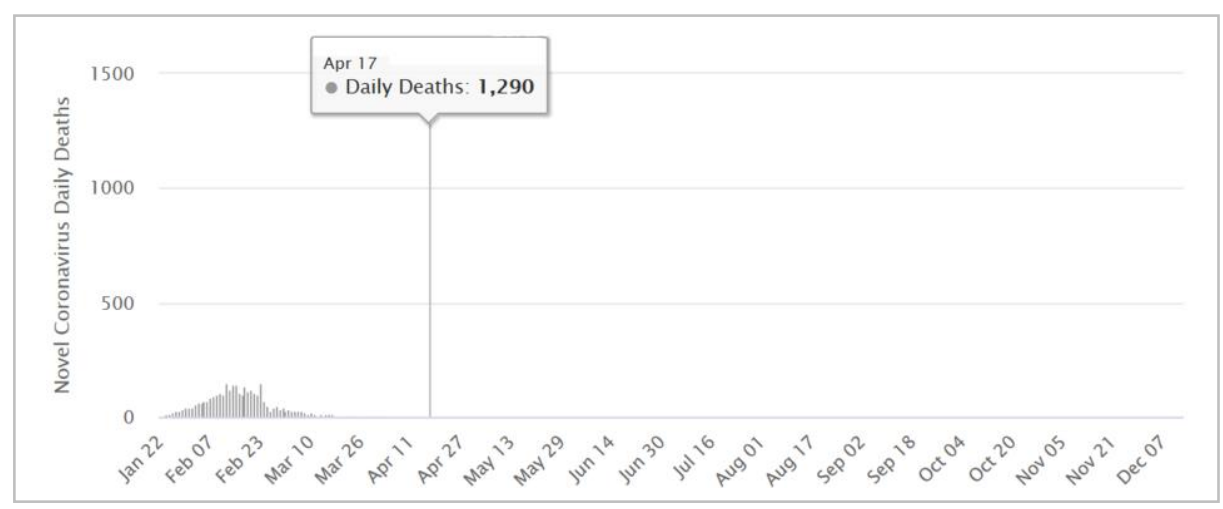

Figure 7. Sudden increase of death number in China on April 17, 2020 [a screenshot, on Dec 10, 2020].

In some cases a sharp spike of deaths in the daily mortality chart reflected a real increase mortality in the certain day (Fig. 8), that was in Georgia on April 7, 2020: "April 7 worst virus day so far in Georgia; big increases in cases, deaths, hospitalizations". ${ }^{21}$

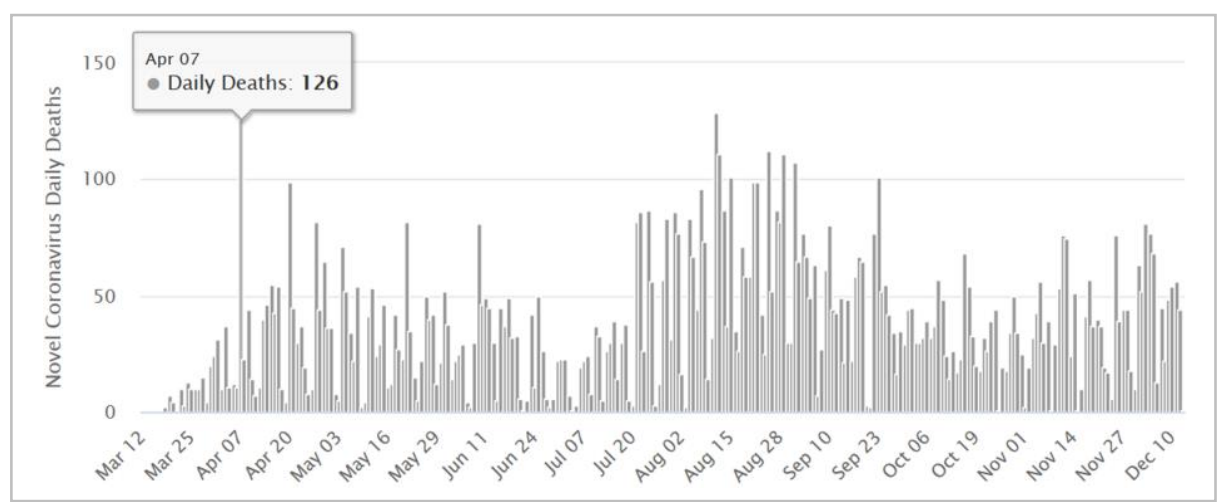

Figure 8. Sudden increase of death number in Georgia on April 7, 2020 [a screenshot, on December 10, 2020].

There was also an opposite correction that took place in France: a number of CoViD-19 victims was decreased by 217 cases on May 19, 2020 (Fig 9). ${ }^{21}$

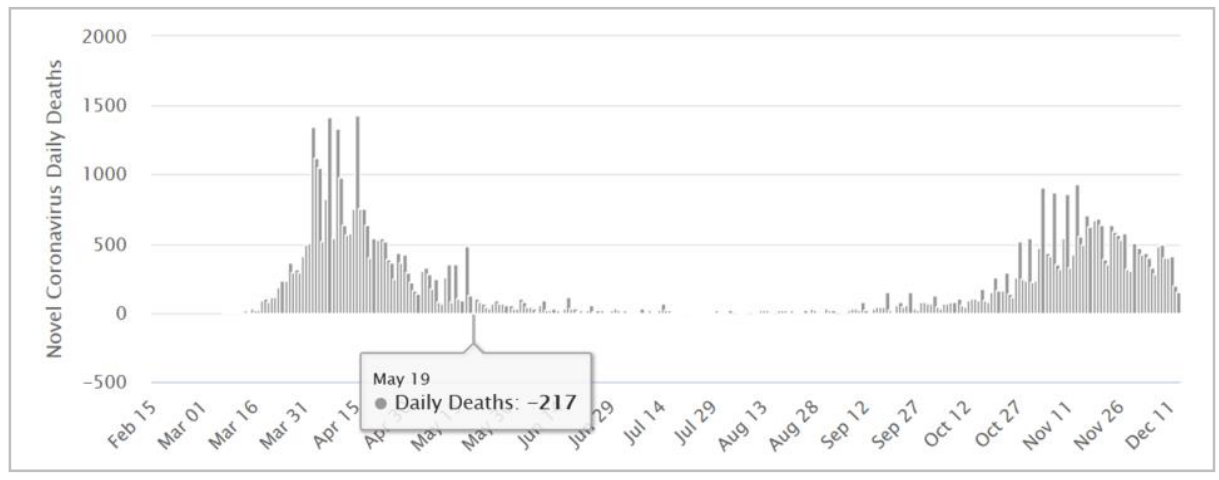

Figure 9. In France a number of CoViD-19 victims was decreased by 217 cases on May 19, 2020 [a screenshot, on December 10, 2020]. 


\section{Updating Global Mortality Data and Weekly Mortality Cycle related to CoViD-19.}

Background: During the current observation death numbers on the 'Worldometer' website related to the whole world and to the various countries were updating constantly.

Objective: To study if updating and correction of the mortality data affects the global weekly mortality cycle related to CoViD-19.

8.1. Method: In order to know how update of the database affects the absolute values of the total global mortality and mortality on the various days of the week, a comparison of death numbers for the first 23 weeks of pandemic (26.01.20-04.07.20) obtained at the different dates, has been done (Tab. 9).

Table 9. Global Mortality Due to CoViD-19 on Various Days of the Week (26.01.20 - 04.07.20).

There are absolute values, $\pm \Delta$ and HLM ratio. Data were obtained on different dates.

\begin{tabular}{|r|r|r|r|r|r|r|r|r|r|r|}
\hline Date & Total & $\pm \Delta$ & Sun & Mon & Tue & Wed & Thu & Fri & Sat & HLM \\
\hline 06.07 & 532,817 & n/a & 57,351 & 60,200 & 84,380 & 85,075 & 85,151 & 84,617 & 76,043 & 1.485 \\
\hline 12.07 & 532,972 & +155 & 57,360 & 60,208 & 84,401 & 85,093 & 85,181 & 84,641 & 76,088 & 1.485 \\
\hline 02.08 & 536,657 & $+3,685$ & 57,872 & 60,643 & 84,891 & 85,679 & 85,674 & 85,226 & 76,672 & 1.480 \\
\hline 06.08 & 537,221 & +564 & 57,947 & 60,712 & 84,954 & 85,740 & 85,801 & 85,304 & 76,763 & 1.481 \\
\hline 17.08 & 539,314 & $+2,657$ & 58,575 & 61,464 & 85,213 & 85,703 & 86,085 & 85,385 & 76,889 & 1.470 \\
\hline 27.08 & 538,730 & -584 & 58,719 & 61,708 & 84,941 & 85,420 & 86,050 & 84,985 & 76,907 & 1.465 \\
\hline 03.09 & 538,718 & -12 & 58,719 & 61,708 & 84,941 & 85,420 & 86,038 & 84,985 & 76,907 & 1.465 \\
\hline 07.09 & 541,679 & $+2,961$ & 59,242 & 61,935 & 85,405 & 85,723 & 86,357 & 85,641 & 77,376 & 1.458 \\
\hline 15.09 & 541,679 & 0 & 59,242 & 61,935 & 85,405 & 85,723 & 86,357 & 85,641 & 77,376 & 1.458 \\
\hline 20.09 & 541,709 & +30 & 59,244 & 61,936 & 85,411 & 85,729 & 86,362 & 85,645 & 77,382 & 1.458 \\
\hline 20.10 & 545,031 & $+3,322$ & 59,621 & 62,457 & 85,787 & 86,117 & 86,964 & 86,183 & 77,902 & 1.459 \\
\hline 20.11 & 546,394 & $+1,363$ & 59,817 & 62,613 & 85,917 & 86,394 & 87,152 & 86,391 & 78,110 & 1.457 \\
\hline 15.12 & 546,547 & +153 & 59,831 & 62,624 & 85,934 & 86,414 & 87,177 & 86,414 & 78,153 & 1.457 \\
\hline
\end{tabular}

Results: During the current study the total numbers of deaths related to the time frame between January 26 and July 4, 2020, were not stable. There was a fluctuation of the death numbers in the various days of the week, nevertheless the lowest numbers of deaths were on Sunday and the highest numbers of deaths were mostly on Thursday. A ratio between the death numbers on Thursday and Sunday decreased from 1.485, on July 6, to 1.457, on December 15, 2020.

8.3. Method: In order to know how update of the database affects the absolute values of the total global mortality and mortality on the various days of the week, an additional comparison of death numbers for other 18 weeks (29.03.20-01.08.20), obtained at the different dates, has been done (Tab. 10).

Results: During the current study the total numbers of deaths related to the time frame between March 29 and August 1, 2020, were not stable. There was a fluctuation of the death numbers in the various days of the week, nevertheless the lowest numbers of death were on Sunday and the highest 
numbers of death were on Wednesday. A ratio between the death numbers on Wednesday and Sunday decreased from 1.497, on August 2, to 1.464, on December 15, 2020.

Table 10. Global Mortality Due to CoViD-19 on Various Days of the Week (29.03.20 - 01.08.20).

There are absolute values, $\pm \Delta$ and HLM ratio. Data were obtained on different dates.

\begin{tabular}{|c|c|r|c|c|c|c|c|c|c|c|}
\hline Date & \multicolumn{1}{|c|}{ Total } & \multicolumn{1}{|c|}{$\pm \Delta$} & \multicolumn{1}{c|}{ Sun } & Mon & Tue & Wed & Thu & Fri & Sat & HLM \\
\hline 02.08 & 655,799 & n/a & 71,540 & 73,337 & 103,569 & 107,080 & 104,963 & 103,286 & 92,024 & 1.497 \\
\hline 06.08 & 656,362 & +563 & 71,615 & 73,406 & 103,632 & 107,141 & 105,089 & 103,364 & 92,115 & 1.496 \\
\hline 17.08 & 655,904 & -458 & 72,038 & 73,964 & 103,294 & 106,701 & 105,038 & 103,032 & 91,837 & 1.481 \\
\hline 27.08 & 654,940 & -964 & 72,086 & 74,127 & 102,928 & 106,335 & 105,270 & 102,532 & 91,662 & 1.475 \\
\hline 03.09 & 654,917 & -23 & 72,084 & 74,127 & 102,929 & 106,334 & 105,255 & 102,528 & 91,660 & 1.475 \\
\hline 07.09 & 658,879 & +3962 & 72,707 & 74,454 & 103,579 & 106,765 & 105,714 & 103,343 & 92,317 & 1.469 \\
\hline 15.09 & 658,879 & 0 & 72,707 & 74,454 & 103,579 & 106,765 & 105,714 & 103,343 & 92,317 & 1.469 \\
\hline 20.09 & 658,938 & +59 & 72,709 & 74,458 & 103,593 & 106,775 & 105,723 & 103,353 & 92,327 & 1.469 \\
\hline 20.10 & 665,569 & +6631 & 73,566 & 75,173 & 104,430 & 107,720 & 106,812 & 104,432 & 93,436 & 1.464 \\
\hline 20.11 & 666,753 & +1184 & 73,749 & 75,300 & 104,526 & 107,998 & 106,964 & 104,617 & 93,599 & 1.464 \\
\hline 15.12 & 666,940 & +187 & 73,772 & 75,319 & 104,550 & 108,013 & 106,990 & 104,642 & 93,654 & 1.464 \\
\hline
\end{tabular}

Conclusion: The fluctuation of the total mortality due to CoViD-19 and mortality on the various days of the week didn't affect the Weekly Mortality Cycle in general. Nevertheless additional study is required to know a reason for decrease and increase of the death numbers related to the past dates.

\section{Updating Global Mortality Data and Weekly Mortality Cycle in various countries.}

Background: During the current observation death numbers on the 'Worldometer' website were updating constantly. In some countries mortality data related to the past were fairly stable, in others, the correction occurred weekly or even every 2-3 days: either up or down.

Objective: To study if the correction of the database for the days related to the past affects the weekly mortality cycle in various countries.

Methods: The database related to mortality due to CoViD-19 in various countries was collected on a regular basis. A comparison of databases collected on August 10 and September 10, 2020 are presented in Tab. 11.

Results: For the majority of countries, mortality data for the past months was stable and did not change over time $[\Delta=0]$. Small changes have occurred in the databases of Belgium [+9], France [-24], Iran [-37], Netherlands [+125], Italy [+152] and the Unites States of America [+284]. Significant changes have been observed in the databases of Peru [+ 4064] and the United Kingdom [- 4925]. 


\section{Table 11.}

Mortality in various days of the week in Brazil, Chile, Mexico, Germany, Russia, the USA and the UK, etc.

For each country, the numbers of death collected on August 10, 2020 are in the first row; the numbers of death collected on September 10, 2020 are in the second row; the difference $(+/-\Delta)$ between two numbers are in the third row.

\begin{tabular}{|c|c|c|c|c|c|c|c|c|}
\hline & otal & Sun & n & Tue & ed & Thu & Fri & Sat \\
\hline ) & $\begin{array}{c}155,115 \\
155,399 \\
+284\end{array}$ & $\begin{array}{l}13,825 \\
13,827 \\
+2\end{array}$ & $\begin{array}{l}16,024 \\
16,028 \\
+4\end{array}$ & $\begin{array}{l}27,178 \\
27,180 \\
+2\end{array}$ & $\begin{array}{l}27,525 \\
\quad 27,525 \\
0\end{array}$ & $\begin{array}{c}25,638 \\
26,015 \\
+377\end{array}$ & $\begin{array}{c}24,302 \\
24,289 \\
-13\end{array}$ & $\begin{array}{l}20,623 \\
20,535 \\
-88\end{array}$ \\
\hline $\begin{array}{l}\text { razil: } \\
6.04-01.08) \\
+/-\end{array}$ & $\begin{array}{r}89,571 \\
0\end{array}$ & $\begin{array}{r}7,398 \\
\quad 7,398 \\
0\end{array}$ & $\begin{array}{l}, 153 \\
\quad 9,153 \\
0\end{array}$ & $\begin{array}{l}15,378 \\
\quad 15,378 \\
0\end{array}$ & $\begin{array}{l}\begin{array}{l}15,161 \\
15,161 \\
0\end{array} \\
\end{array}$ & $\begin{array}{l}15,498 \\
15,498 \\
0\end{array}$ & $\begin{array}{l}14,423 \\
14,423 \\
0\end{array}$ & $\begin{array}{l}12,560 \\
12,560 \\
0\end{array}$ \\
\hline $\begin{array}{l}\text { Mexico: } \\
(19.04-01.08) \\
\Delta+/-\end{array}$ & $\begin{array}{l}46,142 \\
0\end{array}$ & $\begin{array}{l}5,425 \\
\quad 5,425 \\
0\end{array}$ & $\begin{array}{r}3,704 \\
\quad 3,704 \\
0\end{array}$ & $\begin{array}{l}4,598 \\
\quad 4,598 \\
0\end{array}$ & $\begin{array}{l}8,441 \\
\quad 8,441 \\
0\end{array}$ & $\begin{array}{l}8,548 \\
\quad 8,548 \\
0\end{array}$ & $\begin{array}{l}7,847 \\
\quad 7,847 \\
0\end{array}$ & $\begin{array}{r}7,579 \\
\quad 7,579 \\
0\end{array}$ \\
\hline $\begin{array}{l}(29.03-01.08) \\
\Delta+/-\end{array}$ & $\begin{array}{l}44,670 \\
39,745 \\
-4925\end{array}$ & $\begin{array}{l}4,041 \\
3,737 \\
-304 \\
\end{array}$ & $\begin{array}{c}3,646 \\
3,524 \\
-122 \\
\end{array}$ & $\begin{array}{c}8,333 \\
7,187 \\
-1146 \\
\end{array}$ & $\begin{array}{c}7,598 \\
6,745 \\
-853 \\
\end{array}$ & $\begin{array}{c}6,834 \\
6,147 \\
-687 \\
\end{array}$ & $\begin{array}{c}7,723 \\
6,726 \\
-997 \\
\end{array}$ & $\begin{array}{l}6,495 \\
5,679 \\
-816\end{array}$ \\
\hline $\begin{array}{l}\text { dia } \\
0.05-01.08) \\
+/-\end{array}$ & $\begin{array}{l}33,641 \\
0\end{array}$ & $\begin{array}{l}4,348 \\
\quad 4,348 \\
0\end{array}$ & $\begin{array}{l}4,197 \\
\quad 4,197 \\
0\end{array}$ & $\begin{array}{r}4,739 \\
\quad 4,739 \\
0\end{array}$ & $\begin{array}{r}5,312 \\
\quad 5,312 \\
0\end{array}$ & $\begin{array}{l}4,911 \\
\quad 4,911 \\
0\end{array}$ & $\begin{array}{l}5,104 \\
\quad 5,104 \\
0\end{array}$ & $\begin{array}{l}5,030 \\
\quad 5,030 \\
0\end{array}$ \\
\hline $\begin{array}{l}\text { aly } \\
8.03-23.05) \\
+/-\end{array}$ & $\begin{array}{l}32,441 \\
+152,593 \\
\end{array}$ & $\begin{array}{l}4,035 \\
4,054 \\
+19 \\
\end{array}$ & $\begin{array}{l}4,314 \\
\quad 4,335 \\
+21 \\
\end{array}$ & $\begin{array}{l}4,778 \\
\quad 4,801 \\
+23 \\
\end{array}$ & $\begin{array}{l}4,678 \\
\quad 4,699 \\
+21 \\
\end{array}$ & $\begin{array}{l}4,657 \\
4,677 \\
+20 \\
\end{array}$ & $\begin{array}{l}4,993 \\
5,017 \\
+24 \\
\end{array}$ & $\begin{array}{l}4,986 \\
5,010 \\
+24 \\
\end{array}$ \\
\hline $\begin{array}{l}\text { ain } \\
5.03-13.06) \\
+/-\end{array}$ & $\begin{array}{r}28,088 \\
28,088\end{array}$ & $\begin{array}{l}3,797 \\
\quad 3,797 \\
0\end{array}$ & $\begin{array}{l}3,911 \\
\quad 3,911 \\
0\end{array}$ & $\begin{array}{l}4,003 \\
\quad 4,003 \\
0\end{array}$ & $\begin{array}{r}4,378 \\
\quad 4,378\end{array}$ & $\begin{array}{ll} & 4,035 \\
& 4,035 \\
0 & \\
\end{array}$ & $\begin{array}{r}3,986 \\
\quad 3,986 \\
0\end{array}$ & $\begin{array}{r}3,978 \\
\quad 3,978 \\
0\end{array}$ \\
\hline 16.05$)$ & $\begin{array}{r}27,063 \\
\quad 27,039 \\
-24\end{array}$ & $\begin{array}{r}2,325 \\
2,324 \\
-1 \\
\end{array}$ & $\begin{array}{l}3,564 \\
3,561 \\
-3 \\
\end{array}$ & $\begin{array}{l}4,494 \\
\quad 4,490 \\
-4 \\
\end{array}$ & $\begin{array}{l}4,051 \\
4,046 \\
-5 \\
\end{array}$ & $\begin{array}{l}, 148 \\
\quad 5,143 \\
-5\end{array}$ & $\begin{array}{l}, 121 \\
\quad 4,118 \\
-3 \\
\end{array}$ & $\begin{array}{l}3,360 \\
3,357 \\
-3 \\
\end{array}$ \\
\hline eru & $\begin{array}{l}19,387 \\
23,451 \\
+4064 \\
\end{array}$ & $\begin{array}{l}2,593 \\
3,307 \\
+714 \\
\end{array}$ & $\begin{array}{l}2,626 \\
3,298 \\
+672 \\
\end{array}$ & $\begin{array}{l}2,770 \\
3,357 \\
+587 \\
\end{array}$ & $\begin{array}{c}2,825 \\
3,224 \\
+399 \\
\end{array}$ & $\begin{array}{c}2,847 \\
3,464 \\
+617 \\
\end{array}$ & $\begin{array}{c}2,795 \\
3,346 \\
+551 \\
\end{array}$ & $\begin{array}{l}2,931 \\
3,455 \\
+524 \\
\end{array}$ \\
\hline 08) & $\begin{array}{r}16,976 \\
\quad 16,939 \\
-37\end{array}$ & $\begin{array}{l}2,333 \\
2,331 \\
-2\end{array}$ & $\begin{array}{l}2,425 \\
2,421 \\
-4\end{array}$ & $\begin{array}{l}2,451 \\
2,447 \\
-4\end{array}$ & $\begin{array}{l}2,458 \\
2,455 \\
-3\end{array}$ & $\begin{array}{l}2,538 \\
2,531 \\
-7\end{array}$ & $\begin{array}{l}2,365 \\
2,357 \\
-8\end{array}$ & $\begin{array}{l}2,406 \\
2,397 \\
-9\end{array}$ \\
\hline a: & $\begin{array}{r}13,377 \\
13,377\end{array}$ & $\begin{array}{r}1,499 \\
\quad 1,499 \\
0\end{array}$ & $\begin{array}{l}1,414 \\
\quad 1,414 \\
0\end{array}$ & $\begin{array}{l}2,093 \\
\quad 2,093 \\
0\end{array}$ & $\begin{array}{r}2,204 \\
2,204\end{array}$ & \begin{tabular}{ll}
\multicolumn{1}{r|}{1,966} \\
& 1,966 \\
0 & \\
\end{tabular} & $\begin{array}{ll}2,224 \\
\quad 2,224 \\
0\end{array}$ & $\begin{array}{l}1,977 \\
\quad 1,977 \\
0\end{array}$ \\
\hline .08) & $\begin{array}{l}10,230 \\
10,230\end{array}$ & $\begin{array}{l}1,343 \\
\quad 1,343 \\
0\end{array}$ & $\begin{array}{l}1,164 \\
\quad 1,164 \\
0\end{array}$ & $\begin{array}{l}1,356 \\
\quad 1,356 \\
0\end{array}$ & $\begin{array}{r}1,429 \\
1,429\end{array}$ & $\begin{array}{ll}1,694 \\
0 \\
0\end{array}$ & $\begin{array}{l}1,698 \\
\quad 1,698 \\
0\end{array}$ & $\begin{array}{l}1,546 \\
0 \\
0\end{array}$ \\
\hline (22. & $\begin{array}{ll}9,403 & \\
& 9,412 \\
+9 & \end{array}$ & $\begin{array}{c}1,184 \\
1,322 \\
+138\end{array}$ & $\begin{array}{c}1,062 \\
1,315 \\
+253\end{array}$ & $\begin{array}{c}1,225 \\
1,376 \\
+151\end{array}$ & $\begin{array}{c}1,605 \\
1,354 \\
-251\end{array}$ & $\begin{array}{l}1,475 \\
1,447 \\
-28\end{array}$ & $\begin{array}{c}1,559 \\
1,278 \\
-281\end{array}$ & $\begin{array}{l}1,293 \\
1,320 \\
+27\end{array}$ \\
\hline $\begin{array}{l}\text { Chile } \\
(17.05-01.08)\end{array}$ & 8,496 & $\begin{array}{l}1,354 \\
\quad 1,354 \\
0\end{array}$ & $\begin{array}{l}719 \\
0 \\
\end{array}$ & $\begin{array}{l}552 \\
0 \\
\end{array}$ & $\begin{array}{l}1,360 \\
\quad 1,360 \\
0\end{array}$ & $\begin{array}{ll}1,490 \\
& 1,490 \\
0 & \\
\end{array}$ & $\begin{array}{ll}1,448 \\
& 1,448 \\
0 & \\
\end{array}$ & $\begin{array}{l}1,573 \\
\quad 1,573 \\
0\end{array}$ \\
\hline ) & 7,933 & $\begin{array}{l}698 \\
0 \\
\end{array}$ & $\begin{array}{l}1,165 \\
\quad 1,165 \\
0\end{array}$ & $\begin{array}{r}1,337 \\
1,337\end{array}$ & $\begin{array}{r}1,521 \\
1,521\end{array}$ & $\begin{array}{ll} & 1,321 \\
& 1,321 \\
0 & \\
\end{array}$ & $\begin{array}{r}1,129 \\
\quad 1,129 \\
0\end{array}$ & $\begin{array}{l}762 \\
0\end{array}$ \\
\hline $\begin{array}{l}(12 . \\
\Delta+\end{array}$ & 7,615 & $\begin{array}{l}887 \\
0 \\
\end{array}$ & $\begin{array}{l}884 \\
0 \\
\end{array}$ & $\begin{array}{l}1,117 \\
0 \\
0\end{array}$ & $\begin{array}{l}1,158 \\
\quad 1,158 \\
0\end{array}$ & $\begin{array}{l}1,344 \\
0 \\
0\end{array}$ & $\begin{array}{l}1,095 \\
\quad 1,095 \\
0\end{array}$ & $\begin{array}{l}1,130 \\
0 \\
0\end{array}$ \\
\hline $\begin{array}{l}\text { South Africa } \\
(17.05-01.08) \\
\Delta+/-\end{array}$ & $\begin{array}{ll}7,509 & \\
& 7,509 \\
0 & \\
\end{array}$ & $\begin{array}{l}744 \\
0 \\
\end{array}$ & $\begin{array}{l}1,042 \\
\quad 1,042 \\
0\end{array}$ & $\begin{array}{l}1,248 \\
0 \\
0\end{array}$ & $\begin{array}{l}1,020 \\
0 \\
0\end{array}$ & $\begin{array}{r}1,232 \\
1,232\end{array}$ & $\begin{array}{l}1,160 \\
\quad 1,160 \\
0\end{array}$ & $\begin{array}{r}1,063 \\
\quad 1,063 \\
0\end{array}$ \\
\hline $\begin{array}{l}\text { Netherlands } \\
(15.03-20.06) \\
\Delta+/-\end{array}$ & $\begin{array}{r}5,815 \\
+125 \\
+1240\end{array}$ & $\begin{array}{r}641 \\
+849 \\
+8\end{array}$ & $\begin{array}{rr}488 & \\
& 492 \\
+4 & \\
\end{array}$ & $\begin{array}{l}9941,013 \\
+19\end{array}$ & $\begin{array}{r}969 \\
\\
+16 \\
\end{array}$ & $\begin{array}{r}951969 \\
+18 \\
\end{array}$ & $\begin{array}{rr}894 & \\
& 924 \\
+30 & \\
\end{array}$ & $\begin{array}{rr}878 & \\
& 908 \\
+30 & \\
\end{array}$ \\
\hline $\begin{array}{l}\text { China } \\
(26.01-14.03) \\
\Delta+/-\end{array}$ & $\begin{array}{l}3143 \\
0\end{array}$ & $\begin{array}{l}497 \\
0\end{array}$ & $\begin{array}{l}415 \\
0\end{array}$ & $\begin{array}{l}436 \\
0\end{array}$ & $\begin{array}{l}442 \\
0\end{array}$ & $\begin{array}{l}436 \\
0\end{array}$ & $\begin{array}{l}472 \\
0\end{array}$ & $\begin{array}{l}445 \\
0\end{array}$ \\
\hline
\end{tabular}


Conclusion: For patients suffering from CoViD-19, the safest days of the week were mostly Sundays or Mondays. Updating and correction of global and local mortality data didn't affect Weekly Mortality Cycle in general, but affected this cycle in certain countries.

\section{A Weekly Cycle relates to Human Civilization.}

In the 18th century, Antoine-Yves Goguet wrote: "We can consider as the first step that men have taken to obtain a measure of time, was the establishment of the small period of seven days, which bears the name of the week. We see that, from time immemorial, it has been used by almost all peoples, and that the arrangement has been perfectly uniform". ${ }^{23}$

Our contemporary, Joseph Needham, expressed a modern view on the calendar and said, that some of its elements are based on those astronomical cycles which have obvious importance for man, such as the day, the month and the year; others are artificial, such as the week and the subdivisions of the day. ${ }^{24}$ Eviatar Zerubavel, describing the characteristics of the weekly cycle, titled his book "Hidden Rhythms". 25

\section{Discussion.}

On the one hand, a week as a time frame obviously exists, but on the other, there is no natural basis that could explain a weekly cycle.

As described above the weekly mortality cycle with the trend towards decreased mortality on weekends is a unique phenomenon that has been observed among victims of CoViD-19. It has an opposite pattern compared to increased hospital mortality on weekends in the United Kingdom discussed in $2015 .^{26}$

According to the professor Igor A. Gundarov, MD, ${ }^{27}$ the treatment of patients with CoViD-19 is still in the phase of clinical trials and mortality in this group correlates with excessive therapeutic activity, which decreases on weekends. ${ }^{28}$

To understand this statement one should recall the stories on the contradictory efficiency of certain medicines and therapeutic modalities used among patients infected with SARS-CoV-2. For example:

a) Application of Hydroxychloroquine:

On May 22, 2020, an article was published in Lancet where authors "were unable to confirm a benefit of hydroxychloroquine or chloroquine, when used alone or with a macrolide, on in-hospital outcomes for COVID-19. Each of these drug regimens was associated with decreased in-hospital survival and an increased frequency of ventricular arrhythmias when used for treatment of COVID19". ${ }^{29}$ On May 25, 2020, WHO suspended hydroxychloroquine trials for COVID-19. ${ }^{30}$ On June 13, 
2020, Lancet retracted an article mentioned above due to the fact that results presented there could not be proven. ${ }^{31}$ On July 4, 2020, WHO discontinues hydroxychloroquine (and lopinavir/ritonavir) treatment arms for COVID-19. ${ }^{32}$ On December 1, 2020, a group of researches published their study, that included the following conclusion: "Hydroxychloroquine use was associated with a $30 \%$ lower risk of death in COVID-19 hospitalized patients". ${ }^{33}$

\section{b) Application of Corticosteroids / Dexamethasone:}

On March 13, 2020, WHO guidance recommended "Do not routinely give systemic corticosteroids for treatment of viral pneumonia outside clinical trials". ${ }^{34}$ On June 16, 2020, Prof Peter Horby said, that dexamethasone "is the only drug so far that has been shown to reduce mortality - and it reduces it significantly". ${ }^{35}$ It reduced deaths by one-third in patients receiving invasive mechanical ventilation, by one-fifth in patients receiving oxygen without invasive mechanical ventilation, but did not reduce mortality in patients not receiving respiratory support at randomization. ${ }^{36}$ Later conclusion on efficacy of corticosteroids was pessimistic: "There is no evidence that corticosteroids are safe and effective on the treatment of severe acute respiratory infection when COVID-19 disease is suspected". ${ }^{37}$

\section{c) Application of Invasive Mechanical Ventilation:}

According to the study done several years before CoViD-19 pandemic, the highest mortality among the severest cases of patients with Acute Respiratory Distress Syndrome, who were treated with invasive mechanical ventilation, did not exceed $50.4 \% .^{38}$

In the very beginning of CoViD-19 pandemic mortality among CoViD-19 patients who were treated with invasive mechanical ventilation was higher than $80 \%$, including, $81 \%,{ }^{39} 88.1 \%$, ${ }^{40}$ $92 \%,{ }^{41}$ and $97 \% .{ }^{42}$ One may suppose that fatal outcome which is higher than $68 \%$ means a standard one, ${ }^{43}$ so although invasive mechanical ventilation is still in use, it has no therapeutic effect among patients with Acute Respiratory Distress Syndrome caused by SARS-CoV-2.

\section{Conclusion.}

It is vitally important to recognize factors which formed the basis of decreased mortality on Sundays, Mondays and some other "safest days" of the week. These factors can relate to various aspects of healthcare, including therapeutic protocols and work schedules, or to human habits and traditions. Presumably, they relate to the optimised therapeutic protocols used on the "safest days".

If the factors, which reduced mortality on certain days of the week, could be identified, their positive effect should be spread to other days of the week. As a result many lives of CoViD-19 patients could be saved in future.

\section{Disclosure Statement:}

The author declares there are no conflicts of interest in the submitted manuscripts. 


\section{Acknowledgment:}

I am grateful to my colleagues from Brazil, China, Italy, Malaysia, and the USA who shared with me there personal experiences and testimonies regarding therapeutic protocols used at their hospitals for CoViD-19 patients.

\section{References:}

1. Wuhan Municipal Health Commission announces pneumonia epidemic. - Dec 31, 2019. [cited 2020 Dec 7]. Available from: https://crofsblogs.typepad.com/h5n1/2019/12/wuhan-municipal-healthcommission-announces-pneumonia-epidemic.html

2. Pneumonia of unknown cause - China, 5 January, 2020. [cited 2020 Dec 7]. Available from: https://www.who.int/csr/don/05-january-2020-pneumonia-of-unkown-cause-china/en/

3. Tan WJ, Zhao XA, Ma XJ, et al. A Novel Coronavirus Genome Identified in a Cluster of Pneumonia Cases - Wuhan, China 2019-2020. - China CDC Weekly, Jan 21, 2020. [cited 2020 Dec 7]. Available from: http://weekly.chinacdc.cn/en/article/id/a3907201-f64f-4154-a19e-4253b453d10c

4. Novel Coronavirus (2[cited 2020 Dec 7]. Available from 019-nCoV). Situation Report - 22. WHO: 11 February 2020. [cited 2020 Dec 7]. Available from: https://www.who.int/docs/defaultsource/coronaviruse/situation-reports/20200211-sitrep-22-ncov.pdf?sfvrsn=fb6d49b1_2

5. WHO Director-General's opening remarks at the media briefing on COVID-19. - WHO, 11 Mar 2020 [cited 2020 Dec 7]. Available from: https://www.who.int/dg/speeches/detail/who-directorgeneral-s-opening-remarks-at-the-media-briefing-on-covid-19---11-march-2020

6. Brown R. Public health lessons learned from biases in coronavirus mortality overestimation. Disaster Med Public Health Prep, 2020, 1-24. doi:10.1017/dmp.2020.298

7. Imai N, Dorigatti I, Cori A, et al. Report 1 - Estimating the potential total number of novel Coronavirus cases in Wuhan City, China - Imperial College London, January 17, 2020. [cited 2020 Dec 7]. Available from; https://www.imperial.ac.uk/mrc-global-infectious-disease-analysis/covid19/report-1-case-estimates-of-covid-19/

8. Imai N, Dorigatti I, Cori A, et al. Report 2 - Estimating the potential total number of novel Coronavirus (2019-nCoV) cases in Wuhan City, China. - Imperial College London, January 22, 2020. [cited 2020 Dec 7]. Available from: https://www.imperial.ac.uk/mrc-global-infectious-diseaseanalysis/covid-19/report-2-update-case-estimates-covid-19/

9. Ferguson NM, Laydon D, Nedjati-Gilani G. et al. Report 9 - Impact of non-pharmaceutical interventions (NPIs) to reduce COVID-19 mortality and healthcare demand. Imperial College London, March 16, 2020 [cited 2020 Dec 7]. Available from: https://www.imperial.ac.uk/mrc-global-infectiousdisease-analysis/covid-19/report-9-impact-of-npis-on-covid-19/ 
10. Salje H, Kiem CT, Lefrancq N. et al. Estimating the burden of SARS-CoV-2 in France. Science; 2020, 369(6500): 208-11; DOI: 10.1126/science.abc3517

11. Fenton N, Osman M, Neil M, McLachlan S. Study suggests more people have had coronavirus than previously estimated. - Medicalxpress, June 26, 2020. [cited 2020 Aug 10]. Available from: https://medicalxpress.com/news/2020-06-people-coronavirus-previously.html

12. Worldometers: Coronavirus Worldwide Graphs [cited 2020, Jul 6 - .. - Dec 15]. Available from: https://www.worldometers.info/coronavirus/worldwide-graphs/

13. Worldometers: Reported Cases and Deaths by Country, Territory, or Conveyance. [cited 2020, Jul $6-\ldots$ - Dec 15]. Available from: https://www.worldometers.info/coronavirus/\#countries

14. Ricon-Becker I, Tarrasch R, Blinder P, Ben-Eliyahu S. A seven-day cycle in COVID-19 infection and mortality rates: Are inter-generational social interactions on the weekends killing susceptible people? - medRxiv, May 08, 2020 doi: https://doi.org/10.1101/2020.05.03.20089508

15. Worldometers: Reported Cases and Deaths by Country, Territory, or Conveyance: United States [cited 2020 Jul 6 -...-Dec 15]. Available from: https://www.worldometers.info/coronavirus/country/us/

16. Worldometers: Reported Cases and Deaths by Country, Territory, or Conveyance: China [cited 2020 Dec 15]. Available from: https://www.worldometers.info/coronavirus/country/china/

17. Chinese mainland reports 82,692 overall confirmed COVID-19 cases as Wuhan revises figures: official. - Xinhua, April 17, 2020. [cited 2020 Aug 10]. Available from: http://en.people.cn/ n3/2020/0417/c90000-9681219.html

18. Shah M. Canada approaches $91 \mathrm{~K}$ coronavirus cases; sharp rise in daily deaths due to glitch. Global News, May 31, 2020. [cited 2020 Aug 10]. Available from: https://globalnews.ca/news/7009672 /canada-coronavirus-cases-may-31/

19. Natalia A. Ramos Miranda. Chile Coronavirus Death Toll Jumps Sharply After New Cases Added. - U.S. News, June 7, 2020. [cited 2020 Dec 10]. Available from: https://www.usnews.com/ news/world/articles/2020-06-07/chile-coronavirus-death-toll-jumps-sharply-after-new-cases-added

20. The Latest: India adds over 2,000 deaths as new cases soar. - CityNews (The Associated Press, Jun 16, 2020. [cited 2020 Dec 10]. Available from: https://www.660citynews.com/2020/06/16/ the-latest-india-adds-over-2000-deaths-as-new-cases-soar/

21. Beverly C. April 7 worst virus day so far in Georgia; big increases in cases, deaths, hospitalizations. - The Cytizen, Apr 7, 2020. [cited 2020 Dec 10]. Available from: https://thecitizen.com/2020/04/07/ april-7-worst-virus-day-so-far-in-georgia-big-increases-in-cases-deaths-hospitalizations/

22. France revises COVID-19 toll slightly downwards to 28,022. - Reuters, May 20, 2020. [cited 2020 Dec 10]. Available from: https://www.reuters.com/article/health-coronavirus-france-asualties/ france-revises-covid-19-toll-slightly-downwards-to-28022-idUSP6N2BQ03Y 
23. Goguet A.-Y. De L'Origine des Loix, des Arts, et des Sciences: et de leurs progrès chez les anciens peuples. T 1. - La Haye: Pierre Gosse Junior, 1758: 474-475.

24. Needham J. Calendrical and Planetary Astronomy. - In: Needham J, Ling W. Science and Civilization in China. V 3: Mathematics and the Sciences of the Heavens and the Earth. - Cambridge: Cambridge University Press, 1959: 390-408.

25. Zerubavel E. Hidden Rhythms: Schedules and Calendars in Social Life. - Berkeley: University of California Press, 1985.

26. Aylin P. Making sense of the evidence for the "weekend effect". - BMJ, 2015, Sep 5; 351:h4652. DOI: $10.1136 / \mathrm{bmj} . \mathrm{h} 4652$

27. Igor A. Gundarov, a Doctor of Medicine, professor, member of the Russian Academy of Natural Sciences, candidate of philosophical sciences, a specialist in the field of epidemiology and medical statistics, etc. [cited 2020 Dec 10]. Available from: https://en.wikipedia.org/wiki/Igor_Gundarov

28. Gundarov IA. When the treatment is worse than the disease. - Studio "Rubezh", 2020, Nov 23. [cited 11.12.20], Available from: https://www.youtube.com/watch?v=UnYbpK_3V90\&t=19s

29. Mehra MR, Desai SS, Ruschitzka F, Patel AN. Hydroxychloroquine or chloroquine with or without a macrolide for treatment of COVID-19: a multinational registry analysis. - Lancet, Published online May 22, 2020 https://doi.org/10.1016/S0140-6736(20)31180-6.

30. WHO Director-General's opening remarks at the media briefing on COVID-19. - WHO, 25 May 2020. - [cited 2020 Dec 10]. Available from: https://www.who.int/director-general/speeches/ detail/who-director-general-s-opening-remarks-at-the-media-briefing-on-covid-19---25-may-2020

31. Retraction. [Ref. Mehra MR, et al. Hydroxychloroquine or chloroquine with or without a macrolide for treatment of COVID-19: a multinational registry analysis]. Lancet, 2020, Jun 13, 395(10240): Published on line June 05, 2020; DOI:https://doi.org/10.1016/S0140-6736(20)31324-6

32. WHO discontinues hydroxychloroquine and lopinavir/ritonavir treatment arms for COVID19. - WHO, 4 July, 2020. [cited 2020 Dec 10]. Available from: https://www.who.int/news/item/04-072020-who-discontinues-hydroxychloroquine-and-lopinavir-ritonavir-treatment-arms-for-covid-19

33. [A group of authors, no names] Use of hydroxychloroquine in hospitalised COVID-19 patients is associated with reduced mortality: Findings from the observational multicentre Italian CORIST study. - Eur. J Intern. Med. 2020, Dec 1, 82: 38-47. DOI:https://doi.org/10.1016/j.ejim.2020.08.019

34. Adjunctive therapies for COVID-19: corticosteroids. - In: Clinical management of severe acute respiratory infection (SARI) when COVID-19 disease is suspected. Interim guidance. WHO, 13 March 2020, p. 10.

35. Roberts M. Coronavirus: Dexamethasone proves first life-saving drug. - BBC News online. 16 June 2020; https://www.bbc.com/news/health-53061281; 
36. Horby P, Lim WS, Emberson J, et al. Effect of Dexamethasone in Hospitalized Patients with COVID-19: Preliminary Report. - medRxiv 2020.06.22.20137273; doi: https://doi.org/10.1101/ 2020.06.22.20137273

37. Yousefifard M, Mohamed Ali K, Aghaei A, et al. Corticosteroids on the Management of Coronavirus Disease 2019 (COVID-19): A Systemic Review and Meta-Analysis. Iran J Public Health. 2020 Aug; 49(8):1411-1421. doi: 10.18502/ijph.v49i8.3863.

38. Bellani G, Laffey JG, Pham T, et. al. Epidemiology, Patterns of Care, and Mortality for Patients with Acute Respiratory Distress Syndrome in Intensive Care Units in 50 Countries. JAMA. 2016, Feb 23; 315(8):788-800. DOI: 10.1001/jama.2016.0291

39. Hua J, Qian CC, Luo ZB, Li Q, Wang FL. Invasive mechanical ventilation in COVID-19 patient management: the experience with 469 patients in Wuhan. - Crit Care. 2020, Jun 16, 24: 348. doi: 10.1186/s13054-020-03044-9

40. Richardson S, Hirsch JS, Narasimhan M, et al. Presenting Characteristics, Comorbidities, and Outcomes Among 5700 Patients Hospitalized With COVID-19 in the New York City Area. JAMA, (2020) 323(20), 2052-2059. https://doi.org/10.1001/jama.2020.6775

41. Yang X, Yu Y, Xu J, et al. Clinical course and outcomes of critically ill patients with SARSCoV-2 pneumonia in Wuhan, China: a single-centered, retrospective, observational study. - Lancet Respir Med 2020; published online Feb 21. https://doi.org/10.1016/S2213-2600(20) 30079-5.

42. Zhou F, Yu T, Du RH, et al. Clinical course and risk factors for mortality of adult inpatients with COVID-19 in Wuhan, China: a retrospective cohort study. - Lancet, 2020, Mar 28, 395(10229): 1054-1062. DOI:https://doi.org/10.1016/S0140-6736(20)30566-3

43. Jekel JF, et al. Describing Variation in Data. - In: Jekel JF, Elmore JG, Katz DL. Epidemiology, Biostatistics, and Preventive Medicine. - Philadelphia, etc.: W.B. Sounders Company, 1996: 107-122. 